This is an Open Access article, distributed under the terms of the Creative Commons Attribution licence (http://creativecommons.org/licenses/by/4.0/), which permits unrestricted re-use, distribution, and reproduction in any medium, provided the original work is properly cited.

doi:10.1017/etds.2019.12

\title{
The $\beta$-transformation with a hole at 0
}

\author{
CHARLENE KALLE $\dagger$, DERONG KONG + , NIELS LANGEVELD $\dagger$ and WENXIA LI§ \\ $\dagger$ Mathematical Institute, University of Leiden, PO Box 9512, 2300 RA Leiden, \\ The Netherlands \\ (e-mail: kallecccj@math.leidenuniv.nl,n.d.s.langeveld@math.leidenuniv.nl) \\ $\ddagger$ College of Mathematics and Statistics, Chongqing University, Chongqing 401331, \\ PR China \\ (e-mail: derongkong@126.com) \\ $\S$ School of Mathematical Sciences, Shanghai Key Laboratory of PMMP, East China \\ Normal University, Shanghai 200062, PR China \\ (e-mail:wxli@math.ecnu.edu.cn)
}

(Received 20 March 2018 and accepted in revised form 14 January 2019)

Abstract. For $\beta \in(1,2]$ the $\beta$-transformation $T_{\beta}:[0,1) \rightarrow[0,1)$ is defined by $T_{\beta}(x)=$ $\beta x(\bmod 1)$. For $t \in[0,1)$ let $K_{\beta}(t)$ be the survivor set of $T_{\beta}$ with hole $(0, t)$ given by

$$
K_{\beta}(t):=\left\{x \in[0,1): T_{\beta}^{n}(x) \notin(0, t) \text { for all } n \geq 0\right\} .
$$

In this paper we characterize the bifurcation set $E_{\beta}$ of all parameters $t \in[0,1)$ for which the set-valued function $t \mapsto K_{\beta}(t)$ is not locally constant. We show that $E_{\beta}$ is a Lebesgue null set of full Hausdorff dimension for all $\beta \in(1,2)$. We prove that for Lebesgue almost every $\beta \in(1,2)$ the bifurcation set $E_{\beta}$ contains infinitely many isolated points and infinitely many accumulation points arbitrarily close to zero. On the other hand, we show that the set of $\beta \in(1,2)$ for which $E_{\beta}$ contains no isolated points has zero Hausdorff dimension. These results contrast with the situation for $E_{2}$, the bifurcation set of the doubling map. Finally, we give for each $\beta \in(1,2)$ a lower and an upper bound for the value $\tau_{\beta}$ such that the Hausdorff dimension of $K_{\beta}(t)$ is positive if and only if $t<\tau_{\beta}$. We show that $\tau_{\beta} \leq 1-(1 / \beta)$ for all $\beta \in(1,2)$.

Key words: dimension theory, low-dimensional dynamics, symbolic dynamics 2010 Mathematics Subject Classification: 11K55, 26A30, 37B10, 37E05, 37E15 (Primary); 11A63, 68R15, 28D05 (Secondary)

\section{Introduction}

In recent years open dynamical systems, i.e., systems with a hole in the state space through which mass can leak away at every iteration, have received a lot of attention. Typically one 
wonders about the rate at which mass leaves the system and about the size and structure of the set of points that remain, called the survivor set. In [Urb86, Urb87] Urbański considered $C^{2}$-expanding, orientation-preserving circle maps with a hole of the form $(0, t)$. He studied the way in which the topological entropy of such a map restricted to the survivor set changes with $t$. To be more precise, let $g$ be a $C^{2}$-expanding and orientationpreserving map on the circle $\mathbb{R} / \mathbb{Z} \sim[0,1)$. For $t \in[0,1)$ let $K_{g}(t)$ be the survivor set defined by

$$
K_{g}(t):=\left\{x \in[0,1): g^{n}(x) \notin(0, t) \text { for all } n \geq 0\right\} .
$$

Urbański proved that the function $t \mapsto h_{\text {top }}\left(g \mid K_{g}(t)\right)$ is a devil's staircase, where $h_{\text {top }}$ denotes the topological entropy.

Motivated by the work of Urbański, we consider this situation for the $\beta$-transformation. Given $\beta \in(1,2]$, the $\beta$-transformation $T_{\beta}:[0,1) \rightarrow[0,1)$ is defined by $T_{\beta}(x)=\beta x$ (mod 1). When $\beta=2$, we recover the doubling map. In correspondence with [Urb86], set

$$
K_{\beta}(t):=\left\{x \in[0,1): T_{\beta}^{n}(x) \notin(0, t) \text { for all } n \geq 0\right\} .
$$

The survivor set $K_{\beta}(t)$ splits naturally into two pieces, $K_{\beta}(t)=K_{\beta}^{0}(t) \cup K_{\beta}^{+}(t)$, where

$$
\begin{aligned}
& K_{\beta}^{0}(t)=\left\{x \in[0,1): \exists n T_{\beta}^{n}(x)=0 \text { and } T_{\beta}^{k}(x) \notin(0, t) \text { for all } 0 \leq k<n\right\}, \\
& K_{\beta}^{+}(t)=\left\{x \in[0,1): T_{\beta}^{n}(x) \geq t \text { for all } n \geq 0\right\} .
\end{aligned}
$$

The set $K_{\beta}^{+}(t)$ occurs in Diophantine approximation. Indeed, consider the set

$$
F_{\beta}(t):=\left\{x \in[0,1) \mid T_{\beta}^{n}(x) \geq t \text { for all but finitely many } n \in \mathbb{N}\right\}
$$

of points $x \geq 0$ such that 0 is badly approximable by its orbit under $T_{\beta}$. Then $F_{\beta}(t)$ can be written as a countable union of affine copies of $K_{\beta}^{+}(t)$ and thus $\operatorname{dim}_{H} F_{\beta}(t)=$ $\operatorname{dim}_{H} K_{\beta}^{+}(t)$ for all $t \in[0,1)$. The approximation properties of $\beta$-expansions have been studied by several authors. In [LPWW14] the authors considered the Hausdorff dimension of the set of values $\beta>1$ for which the orbit of 1 approaches a given target value $x_{0}$ at a given speed. This work generalized that of [PS08], where $x_{0}=0$ and the speed is fixed. Other results on the Diophantine approximation properties of $\beta$-expansions can be found in [Nil09, BW14, Cao14, GL15, LW16] among others.

Note that the set-valued map $\epsilon \mapsto K_{\beta}(\epsilon)$ is weakly decreasing. Further on, we show that this map is locally constant almost everywhere, i.e., for almost all $t \in[0,1)$ there exists a $\delta>0$ such that $K_{\beta}(\epsilon)=K_{\beta}(t)$ for all $\epsilon \in[t-\delta, t+\delta]$. Such a result was also obtained by Urbański in [Urb86] for $C^{2}$-expanding circle maps. This fact motivates the study of the right set valued bifurcation set (simply called bifurcation set) $E_{\beta}$ containing all parameters $t \in[0,1)$ such that the set-valued map $\epsilon \mapsto K_{\beta}(\epsilon)$ is not locally constant on any right-sided neighbourhood of $t$, i.e.,

$$
E_{\beta}:=\left\{t \in[0,1): K_{\beta}(\epsilon) \neq K_{\beta}(t) \text { for any } \epsilon>t\right\} .
$$

The local structure of the sets $K_{2}(t)$ and $E_{2}$ was investigated in detail in [Urb86, Nil09]. The following results can be found more or less explicitly in [Urb86]. More recently it was shown in [Nil09] that these properties could also be dealt with using more elementary combinatorial methods. 

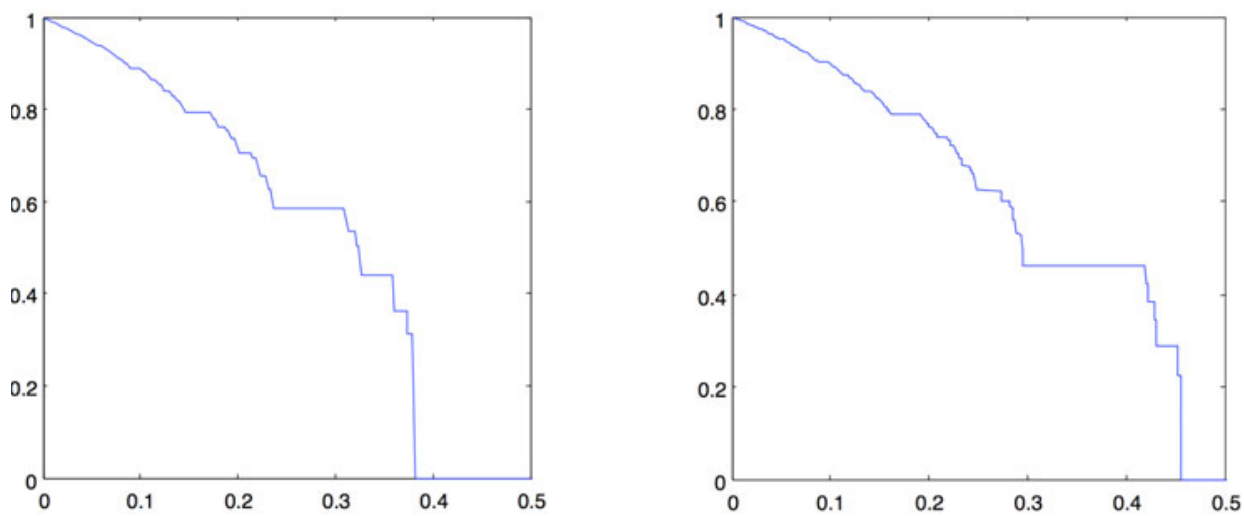

FIGURE 1. Left: the numerical plot of $\eta_{\beta}$ with $\beta \approx 1.61803$ the golden ratio. Right: the numerical plot of $\eta_{\beta}$ with $\beta \approx 1.83929$ the tribonacci number.

\section{THEOREM 1.1. (Urbański [Urb86])}

(i) The bifurcation set $E_{2}$ is a Lebesgue null set of full Hausdorff dimension.

(ii) The function $\eta_{2}: t \mapsto \operatorname{dim}_{H} K_{2}(t)$ is a devil's staircase:

- $\quad \eta_{2}$ is decreasing and continuous on $\left[0, \frac{1}{2}\right]$;

- $\eta_{2}^{\prime}(t)=0$ for Lebesgue almost every $t \in\left[0, \frac{1}{2}\right]$;

- $\eta_{2}(0)=1$ and $\eta_{2}\left(\frac{1}{2}\right)=0$.

(iii) The topological closure $\overline{E_{2}}$ is a Cantor set.

(iv) $\eta_{2}(t)>0$ if and only if $t<\frac{1}{2}$.

Other results on survivor sets for the doubling map $T_{2}$ can be found in e.g. [BY11, Det13, AB14, Sid14, GS15, CT17].

An important ingredient for the proofs in [Urb86, CT17] is the fact that

$$
E_{2}=\left\{t \in[0,1): T_{2}^{n}(t) \geq t \text { for all } n \geq 0\right\} .
$$

This identity does not hold in general for $1<\beta<2$. Therefore, we define $E_{\beta}^{+}$by

$$
E_{\beta}^{+}:=\left\{t \in[0,1): T_{\beta}^{n}(t) \geq t \text { for all } n \geq 0\right\} .
$$

Note that $E_{\beta}^{+} \subseteq E_{\beta}$ but in general these sets do not coincide. In this paper we consider the survivor set $K_{\beta}(t)$ and the bifurcation set $E_{\beta}$ for $\beta \in(1,2)$. We give a detailed description of the topological structure of $E_{\beta}$ and $E_{\beta}^{+}$and their dependence on $\beta$. Theorems A to D below list our main results. Our first result strengthens (i) and (ii) of Theorem 1.1.

THEOREM A. Let $\beta \in(1,2]$ and $t \in[0,1)$.

(i) The bifurcation sets $E_{\beta}$ and $E_{\beta}^{+}$are Lebesgue null sets of full Hausdorff dimension.

(ii) The dimension function $\eta_{\beta}: t \mapsto \operatorname{dim}_{H} K_{\beta}(t)$ is a devil's staircase:

- $\quad \eta_{\beta}$ is decreasing and continuous in $[0,1)$;

- $\eta_{\beta}^{\prime}=0$ Lebesgue almost everywhere in $[0,1)$;

- $\eta_{\beta}$ is not constant.

Figure 1 shows numerical plots of the dimension functions $\eta_{\beta}$ for $\beta \approx 1.61803$, the golden ratio, i.e., the real root bigger than 1 of the polynomial $x^{2}-x-1$ and for 
$\beta \approx 1.83929$, the tribonacci number, i.e., the real root bigger than 1 of the polynomial $x^{3}-x^{2}-x-1$.

The analogous statements of (iii) and (iv) of Theorem 1.1 for $\beta \in(1,2)$ do not always hold. The next main theorems show that in general the topological structure of $E_{\beta}$ differs from that of $E_{2}$ and that this structure depends on the value of $\beta$. Theorems $\mathrm{B}$ and $\mathrm{C}$ imply that (iii) of Theorem 1.1 holds only for a very small set of $\beta \in(1,2)$.

THEOREM B. For Lebesgue almost every $\beta \in(1,2)$ the bifurcation sets $E_{\beta}$ and $E_{\beta}^{+}$ contain infinitely many isolated and accumulation points arbitrarily close to zero and hence their closures are not Cantor sets. On the other hand,

$$
\operatorname{dim}_{H}\left(\left\{\beta \in(1,2): \exists \delta>0 \text { such that } \overline{E_{\beta}^{+}} \cap[0, \delta] \text { is a Cantor set }\right\}\right)=1 .
$$

There are also infinitely many $\beta \in(1,2]$ such that $\overline{E_{\beta}^{+}}$is a Cantor set. This is true, for example, for the countable family of multinacci numbers. In terms of Hausdorff dimension this set is small.

THEOREM C. We have $\operatorname{dim}_{H}\left(\left\{\beta \in(1,2): \overline{E_{\beta}^{+}}\right.\right.$is a Cantor set $\left.\}\right)=0$.

In [Cla16] Clark considered the $\beta$-transformation and characterized the holes of the form $(a, b)$ for which the survivor set $K_{\beta}((a, b))$ is uncountable or not. From the properties of $\eta_{\beta}$ given in Theorem A it follows that for each $\beta \in(1,2]$ there is a unique value $\tau_{\beta}$ such that $\operatorname{dim}_{H} K_{\beta}(t)>0$ if and only if $t<\tau_{\beta}$. By (iv) of Theorem 1.1 we know that $\tau_{2}=1 / 2$. We have the following result on $\tau_{\beta}$.

THEOREM D. For each $\beta \in(1,2]$ we have $\tau_{\beta} \leq 1-(1 / \beta)$ and $\tau_{\beta}=1-(1 / \beta)$ if and only if $\overline{E_{\beta}^{+}}$is a Cantor set.

In [Nil07] Nilsson studied the critical value $\tilde{\tau}_{\beta}$ for the $\beta$-transformation with holes of the form $(t, 1)$. In [Nil07, Proposition 7.12] he proved that for each $\beta \in(1,2), \tilde{\tau}_{\beta}=$ $1-(1 / \beta)$. Many of the proofs use the symbolic codings of the open systems $T_{\beta}$ with hole $(t, 1)$. The main difficulty that we had to overcome in order to extend the results from the doubling map to the $\beta$-transformation is that the $\beta$-transformation is not coded by the full shift on two symbols. In fact, for most values of $\beta$, the associated symbolic system is not even sofic. This might also explain the difference between the result from Theorem $\mathrm{D}$ and the result from [Nil07, Proposition 7.12].

The paper is arranged as follows. In $\S 2$ we introduce some notation, we recall some basic properties of $\beta$-expansions and prove Theorem A. In $\S 3$ we consider the topological structure of $E_{\beta}$ and $E_{\beta}^{+}$and prove Theorem B. By means of Lyndon words we construct infinitely many nested basic intervals which cover the interval $(1,2)$ up to a Lebesgue null set. We can determine all isolated points of $E_{\beta}^{+}$by determining in which intervals it falls. The largest of these intervals are then associated to Farey words, the properties of which allow us to prove Theorem $\mathrm{C}$ in $\S 4$ and Theorem $\mathrm{D}$ in $\S 5$. 
2. Preliminaries, $\beta$-expansions and first properties of $K_{\beta}(t)$ and $E_{\beta}$

In this section we introduce some notation about sequences that is used throughout the paper. We will recall some basic properties of $\beta$-transformations and give some basic results on $K_{\beta}(t)$ and $E_{\beta}$. We also prove Theorem A.

2.1. Notation on sequences. Let $\{0,1\}^{\mathbb{N}}$ be the set of sequences of 0 's and 1 's and let $\sigma$ be the left shift on $\{0,1\}^{\mathbb{N}}$ defined by $\sigma\left(\left(x_{i}\right)\right)=\left(x_{i+1}\right)$. We use $\{0,1\}^{*}$ to denote the set of all finite strings of elements from $\{0,1\}$, called words. A word $w \in\{0,1\}^{n}$ is called a prefix of a sequence $\left(x_{i}\right) \in\{0,1\}^{\mathbb{N}}$ if $x_{1} \ldots x_{n}=w$. For a word $w=w_{1} \ldots w_{n} \in\{0,1\}^{*}$ we write $w^{+}:=w_{1} \ldots w_{n-1}\left(w_{n}+1\right)$ if $w_{n}=0$ and we write $w^{-}:=w_{1} w_{2} \ldots w_{n-1}$ $\left(w_{n}-1\right)$ if $w_{n}=1$. Furthermore, we use $\bar{w}$ to denote the reflection word $\bar{w}:=(1-$ $\left.w_{1}\right)\left(1-w_{2}\right) \ldots\left(1-w_{n}\right)$.

Throughout the paper we use the lexicographical ordering $\prec, \preccurlyeq, \succ$ and $\succcurlyeq$ between sequences and words, which is defined as follows. For two sequences $\left(x_{i}\right),\left(y_{i}\right) \in\{0,1\}^{\mathbb{N}}$ we write $\left(x_{i}\right) \prec\left(y_{i}\right)$ or $\left(y_{i}\right) \succ\left(x_{i}\right)$ if $x_{1}<y_{1}$, or there is an integer $m \geq 2$ such that $x_{i}=y_{i}$ for all $i<m$ and $x_{m}<y_{m}$. Moreover, we say that $\left(x_{i}\right) \preccurlyeq\left(y_{i}\right)$ or $\left(y_{i}\right) \succcurlyeq\left(x_{i}\right)$ if $\left(x_{i}\right) \prec\left(y_{i}\right)$ or $\left(x_{i}\right)=\left(y_{i}\right)$. This definition can be extended to words in the following way. For $u, v \in$ $\{0,1\}^{*}$ we write $u \prec v$ if and only if $u 0^{\infty} \prec v 0^{\infty}$.

Let $\# A$ denote the cardinality of the set $A$. For a subset $\mathcal{Y} \subseteq\{0,1\}^{\mathbb{N}}$ let $\mathcal{B}_{n}(\mathcal{Y})$ denote the set of all words of length $n$ that occur in a sequence in $\mathcal{Y}$. The topological entropy of $\mathcal{Y}$ is then given by

$$
h(\mathcal{Y}):=\lim _{n \rightarrow \infty} \frac{\log \# \mathcal{B}_{n}(\mathcal{Y})}{n}=\inf _{n} \frac{\log \# \mathcal{B}_{n}(\mathcal{Y})}{n},
$$

where the second equality holds since by the definition of $\mathcal{B}_{n}(\mathcal{Y})$ the sequence $\left(\log \# \mathcal{B}_{n}(\mathcal{Y})\right)$ is subadditive. Here and throughout the paper we will use the base-2 logarithm.

2.2. The $\beta$-transformation and $\beta$-expansions. Now we recall some properties of the $\beta$ transformation. Let $\beta \in(1,2]$ and let the (greedy) $\beta$-transformation $T_{\beta}:[0,1) \rightarrow[0,1)$ be given as in the introduction, i.e., $T_{\beta}(x)=\beta x(\bmod 1)$. It has a unique ergodic invariant measure that is equivalent to the Lebesgue measure (cf. [Rén57]). This measure is the unique measure of maximal entropy with entropy equal to $\log \beta$. For each $x \in[0,1)$ the greedy $\beta$-expansion of $x$, denoted by $b(x, \beta)=\left(b_{i}(x, \beta)\right)$, is the sequence obtained from $T_{\beta}$ by setting for each $i \geq 1$,

$$
b_{i}(x, \beta)= \begin{cases}0 & \text { if } T_{\beta}^{i-1}(x) \in\left[0, \frac{1}{\beta}\right), \\ 1 & \text { if } T_{\beta}^{i-1}(x) \in\left[\frac{1}{\beta}, 1\right) .\end{cases}
$$

The name greedy $\beta$-expansion stems from the fact that it is the lexicographically largest sequence $\left(x_{i}\right) \in\{0,1\}^{\mathbb{N}}$ satisfying

$$
x=\sum_{i \geq 1} \frac{x_{i}}{\beta^{i}}=: \pi_{\beta}\left(\left(x_{i}\right)\right) .
$$

We write $b(1, \beta)$ for the sequence $1 b(\beta-1, \beta)$. 
The set of sequences that occur as greedy $\beta$-expansions for a given $\beta$ can be characterized using quasi-greedy $\beta$-expansions. For each $x \in(0,1]$ the quasi-greedy $\beta$ expansion of $x$ is obtained dynamically by iterating the map $\widetilde{T}_{\beta}:(0,1] \rightarrow(0,1]$ given by

$$
\widetilde{T}_{\beta}(x)= \begin{cases}\beta x & \text { if } x \in\left(0, \frac{1}{\beta}\right], \\ \beta x-1 & \text { if } x \in\left(\frac{1}{\beta}, 1\right] .\end{cases}
$$

The only essential difference between the maps $T_{\beta}$ and $\widetilde{T}_{\beta}$ is the value they take at the point $1 / \beta$. For $x \in(0,1]$ the quasi-greedy $\beta$-expansion $\tilde{b}(x, \beta)=\left(\tilde{b}_{i}(x, \beta)\right)$ is then obtained by setting $\tilde{b}_{i}(x, \beta)=0$ if $0<\widetilde{T}_{\beta}^{i-1}(x) \leq(1 / \beta)$ and $\tilde{b}_{i}(x, \beta)=1$ if $(1 / \beta)<\widetilde{T}^{i-1}(x) \leq 1$. The quasi-greedy $\beta$-expansion of 1 plays a crucial role in what follows. For $\beta \in(1,2]$ write

$$
\alpha(\beta):=\tilde{b}(1, \beta) .
$$

Note that if $b(x, \beta)=b_{1} \ldots b_{n} 0^{\infty}$ with $b_{n}=1$, then $\tilde{b}(x, \beta)=b_{1} \ldots b_{n}^{-} \alpha(\beta)$. On the other hand, if $b(x, \beta)$ does not end with $0^{\infty}$, then $b(x, \beta)=\tilde{b}(x, \beta)$. The following characterization of $\alpha(\beta)$ can be found in [KL07, Theorem 2.3]. Let $\mathcal{Q} \subset\{0,1\}^{\mathbb{N}}$ be the set of sequences $\left(a_{i}\right) \in\{0,1\}^{\mathbb{N}}$ not ending with $0^{\infty}$ and satisfying

$$
a_{n+1} a_{n+2} \ldots \preccurlyeq a_{1} a_{2} \ldots \text { for all } n \geq 0 .
$$

LEMMA 2.1. The map $\beta \mapsto \alpha(\beta)$ is a strictly increasing bijection between the interval $(1,2]$ and the set $\mathcal{Q}$.

For a given $\beta$ the sequence $\alpha(\beta)$ determines the set of all greedy $\beta$-expansions in the following way. Let $\Sigma_{\beta}$ be the set of all greedy $\beta$-expansions of $x \in[0,1)$. Then (cf. [Par60])

$$
\Sigma_{\beta}=\left\{\left(x_{i}\right) \in\{0,1\}^{\mathbb{N}}: \sigma^{n}\left(\left(x_{i}\right)\right) \prec \alpha(\beta) \text { for all } n \geq 0\right\} .
$$

Similarly, let $\widetilde{\Sigma}_{\beta}$ be the set of all quasi-greedy $\beta$-expansions of $x \in(0,1]$. Then

$$
\widetilde{\Sigma}_{\beta}=\left\{\left(x_{i}\right) \in\{0,1\}^{\mathbb{N}}: 0^{\infty} \prec \sigma^{n}\left(\left(x_{i}\right)\right) \preccurlyeq \alpha(\beta) \text { for all } n \geq 0\right\} .
$$

The following result can be found in [Par60] (see also [dVKL16]).

LEMMA 2.2. Let $\beta \in(1,2]$. The map $x \mapsto b(x, \beta)$ is a strictly increasing bijection from $[0,1)$ to $\Sigma_{\beta}$ and is right-continuous with respect to the ordering topology on $\Sigma_{\beta}$.

On the other hand, the map $x \mapsto \tilde{b}(x, \beta)$ is a strictly increasing bijection from $(0,1]$ to $\widetilde{\Sigma}_{\beta}$ and is left-continuous with respect to the ordering topology on $\widetilde{\Sigma}_{\beta}$.

2.3. First properties of $K_{\beta}(t)$ and $E_{\beta}$. Let $t \in[0,1)$ be given. Recall the definitions of the survivor set $K_{\beta}(t)=K_{\beta}^{0}(t) \cup K_{\beta}^{+}(t)$ from (1.1) and (1.2). We define the corresponding symbolic survivor sets as the sets of all greedy $\beta$-expansions of elements in the sets $K_{\beta}(t)$, $K_{\beta}^{0}(t)$ and $K_{\beta}^{+}(t)$, respectively. Lemma 2.2 gives the following descriptions:

$$
\begin{aligned}
\mathcal{K}_{\beta}^{+}(t) & =\left\{\left(x_{i}\right) \in\{0,1\}^{\mathbb{N}}: b(t, \beta) \preccurlyeq \sigma^{n}\left(\left(x_{i}\right)\right) \prec \alpha(\beta) \forall n \geq 0\right\}, \\
\mathcal{K}_{\beta}^{0}(t) & =\left\{\left(x_{i}\right) \in\{0,1\}^{\mathbb{N}}: \exists n \geq 0 \sigma^{n}\left(\left(x_{i}\right)\right)=0^{\infty}\right. \\
& \text { and } \left.b(t, \beta) \preccurlyeq \sigma^{k}\left(\left(x_{i}\right)\right) \prec \alpha(\beta) \forall 0 \leq k<n\right\}, \\
\mathcal{K}_{\beta}(t) & =\mathcal{K}_{\beta}^{+}(t) \cup \mathcal{K}_{\beta}^{0}(t) .
\end{aligned}
$$


We will often switch from $K_{\beta}(t)$ to $\mathcal{K}_{\beta}(t)$ and back. The set $K_{\beta}(t)$ is closed and $T_{\beta}$ is continuous when restricted to $K_{\beta}(t)$. Under the metric $d$ on $\{0,1\}^{\mathbb{N}}$ given by

$$
d\left(\left(x_{i}\right),\left(y_{i}\right)\right)=\beta^{-\inf \left\{n \geq 1: x_{n} \neq y_{n}\right\}}
$$

the map $\pi_{\beta}:\left(\mathcal{K}_{\beta}(t), \sigma\right) \rightarrow\left(K_{\beta}(t), T_{\beta}\right)$ is a topological conjugacy. This gives that

$$
h_{\text {top }}\left(T_{\beta} \mid K_{\beta}(t)\right)=h_{\text {top }}\left(\mathcal{K}_{\beta}(t)\right) .
$$

For the bifurcation set $E_{\beta}$, defined in (1.3), the following description can implicitly be found in [Urb86].

Proposition 2.3. $E_{\beta}=\left\{t \in[0,1): t \in K_{\beta}(t)\right\}$ and thus $E_{\beta} \cap[t, 1) \subseteq K_{\beta}(t)$ for any $t \in$ $(0,1)$.

Proof. For all $t \in(0,1)$ we have $t \notin K_{\beta}(\epsilon)$ for any $\epsilon>t$. Hence, if $t \in K_{\beta}(t)$, then $t \in E_{\beta}$. Suppose that $t \notin K_{\beta}(t)$, i.e., there is an $N \geq 1$ such that $T_{\beta}^{N}(t) \in(0, t)$. By the rightcontinuity of $T_{\beta}^{N}$, there is a $\delta>0$ such that

$$
T_{\beta}^{N}(\epsilon) \in\left(T_{\beta}^{N}(t), \frac{T_{\beta}^{N}(t)+t}{2}\right) \subseteq(0, t) \quad \text { for all } \epsilon \in[t, t+\delta] .
$$

This implies that $K_{\beta}(t) \cap[t, t+\delta]=\emptyset$ and thus $K_{\beta}(t+\delta) \subseteq K_{\beta}(t) \subseteq K_{\beta}(t+\delta)$. We conclude that the function $\epsilon \mapsto K_{\beta}(\epsilon)$ is constant on $[t, t+\delta]$, so $t \notin E_{\beta}$.

COROLlary 2.4. For each $\beta \in(1,2]$ the set $[0,1) \backslash E_{\beta}$ is open.

Proof. Let $t \notin E_{\beta}$. The proof of the previous proposition then gives a $\delta_{1}>0$ such that $\left[t, t+\delta_{1}\right] \cap E_{\beta}=\emptyset$. From $t \notin K_{\beta}(t)$ it follows that there is an $N \geq 1$ such that $T_{\beta}^{N}(t) \in$ $(0, t)$. Hence, $T_{\beta}^{k}(t) \neq(1 / \beta)$ for any $0 \leq k \leq N$, which means that $T_{\beta}^{N}$ is left-continuous in $t$. Then, as in the proof of Proposition 2.3, we can find a $\delta_{2}>0$ such that $\left[t-\delta_{2}, t\right] \cap$ $E_{\beta}=\emptyset$.

In (1.4) the set $E_{\beta}^{+}$was defined. By the same proof as given for Proposition 2.3 we also get that $E_{\beta}^{+}$is the bifurcation set of $K_{\beta}^{+}(t)$, i.e.,

$$
E_{\beta}^{+}=\left\{t \in[0,1): t \in K_{\beta}^{+}(t)\right\}=\left\{t \in[0,1): K_{\beta}^{+}(\epsilon) \neq K_{\beta}^{+}(t) \text { for any } \epsilon>t\right\} .
$$

As for $K_{\beta}(t)$ we add a third set $E_{\beta}^{0}$ of the elements in $E_{\beta}$ that are pre-images of 0:

$$
E_{\beta}^{0}=\left\{t \in E_{\beta}: \exists n \geq 0 T_{\beta}^{n}(t)=0\right\}=\left\{t \in[0,1): t \in K_{\beta}^{0}(t)\right\} .
$$

Then $E_{\beta}=E_{\beta}^{+} \cup E_{\beta}^{0}$ and $E_{\beta}^{+} \cap E_{\beta}^{0}=\{0\}$.

The symbolic bifurcation sets, i.e., the sets of all greedy $\beta$-expansions of elements in $E_{\beta}, E_{\beta}^{+}$and $E_{\beta}^{0}$, can be described as follows:

$$
\begin{aligned}
& \mathcal{E}_{\beta}^{+}=\left\{\left(t_{i}\right) \in\{0,1\}^{\mathbb{N}}: \forall n \geq 0 \quad\left(t_{i}\right) \preccurlyeq \sigma^{n}\left(\left(t_{i}\right)\right) \prec \alpha(\beta)\right\}, \\
& \mathcal{E}_{\beta}^{0}=\left\{\left(t_{i}\right) \in\{0,1\}^{\mathbb{N}}: \exists n \geq 0 \sigma^{n}\left(\left(t_{i}\right)\right)=0^{\infty}\right. \\
& \left.\quad \text { and }\left(t_{i}\right) \preccurlyeq \sigma^{k}\left(\left(t_{i}\right)\right) \prec \alpha(\beta) \text { for all } 0 \leq k<n\right\}, \\
& \mathcal{E}_{\beta}=\mathcal{E}_{\beta}^{+} \cup \mathcal{E}_{\beta}^{0} .
\end{aligned}
$$


In the series of papers [Rai89, Rai92, Rai94], Raith studied invariant sets for piecewise monotone expanding maps on the interval [0,1]. More specifically, in [Rai94] he removed a finite number of open intervals from $[0,1]$ and considered piecewise monotone expanding maps restricted to the survivor set. He then studied the dependence on the end points of the holes of the Hausdorff dimension of the survivor set and of the topological entropy of the map restricted to the survivor set. Since no $x \in[0,1)$ has $T_{\beta}(x)=1$, we can apply these results to $T_{\beta}$ on $[0,1)$ with the single hole $(0, t)$ removed. In particular, applying the results from [Rai94, Corollary 1.1 and Theorem 2] gives the following.

Proposition 2.5. [Rai94] Let $\beta \in(1,2)$ be given. The maps $H_{\beta}: t \mapsto h_{\text {top }}\left(\mathcal{K}_{\beta}(t)\right)$ and $\eta_{\beta}: t \mapsto \operatorname{dim}_{H} K_{\beta}(t)$ are continuous on $[0,1)$.

In the process of proving [Rai94, Theorem 2] Raith proved in [Rai94, Lemma 3] that Bowen's dimension formula also holds in this case, i.e., the Hausdorff dimension of the survivor set is the unique zero of the pressure function. In our setting this translates to the following dimension formula:

$$
\operatorname{dim}_{H} K_{\beta}(t)=\frac{h_{\text {top }}\left(T_{\beta} \mid K_{\beta}(t)\right)}{\log \beta} .
$$

Since for any $t \in[0,1)$ the sets $K_{\beta}^{0}(t)$ and $E_{\beta}^{0}$ contain at most countably many points, we have the following properties for the sets under consideration. Let $\lambda$ denote the onedimensional Lebesgue measure.

\begin{tabular}{ll}
\hline $\operatorname{dim}_{H} K_{\beta}(t)=\operatorname{dim}_{H} K_{\beta}^{+}(t)$ & $\operatorname{dim}_{H} K_{\beta}^{0}(t)=0$ \\
$\lambda\left(K_{\beta}(t)\right)=\lambda\left(K_{\beta}^{+}(t)\right)$ & $\lambda\left(K_{\beta}^{0}(t)\right)=0$ \\
$\operatorname{dim}_{H} E_{\beta}=\operatorname{dim}_{H} E_{\beta}^{+}$ & $\operatorname{dim}_{H} E_{\beta}^{0}=0$ \\
$\lambda\left(E_{\beta}\right)=\lambda\left(E_{\beta}^{+}\right)$ & $\lambda\left(E_{\beta}^{0}\right)=0$ \\
$h_{\text {top }}\left(K_{\beta}(t)\right)=\max \left\{h_{\text {top }}\left(K_{\beta}^{+}(t)\right), h_{\text {top }}\left(K_{\beta}^{0}(t)\right)\right\}$ & \\
\hline
\end{tabular}

This table implies that for Theorem A(i) it is enough to consider only $E_{\beta}$. From Proposition 2.5 and (2.6) we also get that $t \mapsto \operatorname{dim}_{H} K_{\beta}^{+}(t)$ is continuous and that

$$
h_{\text {top }}\left(\mathcal{K}_{\beta}(t)\right)=\operatorname{dim}_{H}\left(K_{\beta}^{+}(t)\right) \log \beta .
$$

The next result specifies the relations between the sets even further.

Proposition 2.6. Let $\beta \in(1,2)$. If $t \in E_{\beta}^{+}$, then $h_{\text {top }}\left(\mathcal{K}_{\beta}(t)\right)=h_{\text {top }}\left(\mathcal{K}_{\beta}^{+}(t)\right)$.

Proof. Since $\mathcal{K}_{\beta}^{+}(t) \subseteq \mathcal{K}_{\beta}(t)$, it suffices to prove that $h_{\text {top }}\left(\mathcal{K}_{\beta}^{+}(t)\right) \geq h_{\text {top }}\left(\mathcal{K}_{\beta}(t)\right)$. For $t=0$ there is nothing to prove. Take $t \in E_{\beta}^{+} \backslash\{0\}$ and write $\left(t_{i}\right):=b(t, \beta)$. Then

$$
\left(t_{i}\right) \preccurlyeq \sigma^{n}\left(\left(t_{i}\right)\right) \prec \alpha(\beta) \text { for all } n \geq 0 .
$$

Hence, $\left(t_{i}\right)$ does not end with $0^{\infty}$ and by (2.4) we can rewrite $\mathcal{K}_{\beta}^{0}(t)$ as

$$
\mathcal{K}_{\beta}^{0}(t)=\left\{\left(x_{i}\right): \exists n \geq 0 \sigma^{n}\left(\left(x_{i}\right)\right)=0^{\infty} \text { and }\left(t_{i}\right) \prec \sigma^{k}\left(\left(x_{i}\right)\right) \prec \alpha(\beta) \forall 0 \leq k<n\right\} .
$$


We claim that

$$
\left|\mathcal{B}_{k}\left(\mathcal{K}_{\beta}^{0}(t)\right)\right| \leq \sum_{j=1}^{k+1}\left|\mathcal{B}_{j-1}\left(\mathcal{K}_{\beta}^{+}(t)\right)\right| .
$$

Take a word $a_{1} \ldots a_{k} \in \mathcal{B}_{k}\left(\mathcal{K}_{\beta}^{0}(t)\right)$ and without loss of generality suppose that it occurs as a prefix of a sequence $\left(x_{i}\right) \in \mathcal{K}_{\beta}^{0}(t)$, i.e., $\left(x_{i}\right)=a_{1} \ldots a_{k} x_{k+1} x_{k+2} \ldots$ Let $j \geq 0$ be such that $x_{j}=1$ and the tail $x_{j+1} x_{j+2} \ldots=0^{\infty}$. If $j=0$, then $\left(x_{i}\right)=0^{\infty}$. Avoiding this trivial case we assume that $j \geq 1$ and we will prove that $x_{1} \ldots x_{j-1} 0 \in \mathcal{B}_{j}\left(\mathcal{K}_{\beta}^{+}(t)\right)$. By (2.7) it follows that

$$
t_{1} \ldots t_{j-i} \preccurlyeq x_{i+1} \ldots x_{j-1} 0 \prec \alpha_{1}(\beta) \ldots \alpha_{j-i}(\beta) \quad \text { for all } 0 \leq i<j .
$$

Let $i^{*} \leq j$ be the smallest index such that $x_{i^{*}+1} \ldots x_{j-1} 0=t_{1} \ldots t_{j-i^{*}}$. If strict inequalities in (2.8) hold for all $i<j$, then we put $i^{*}=j$. Note that $\left(t_{i}\right) \preccurlyeq \sigma^{n}\left(\left(t_{i}\right)\right) \prec \alpha(\beta)$ for all $n \geq 0$. Then by the minimality of $i^{*}$ it follows that

$$
x_{1} \ldots x_{j-1} 0 t_{j-i^{*}+1} t_{j-i^{*}+2} \ldots=x_{1} \ldots x_{i^{*}} t_{1} t_{2} \ldots \in \mathcal{K}_{\beta}^{+}(t) .
$$

Observe that $x_{1} \ldots x_{j-1}=a_{1} \ldots a_{j-1}$ if $j \leq k$ and $x_{1} \ldots x_{k}=a_{1} \ldots a_{k}$ if $j \geq k+1$. This implies that $a_{1} \ldots a_{j-1}=x_{1} \ldots x_{j-1} \in \mathcal{B}_{j-1}\left(\mathcal{K}_{\beta}^{+}(t)\right)$ if $j \leq k$ or $a_{1} \ldots a_{k} \in$ $\mathcal{B}_{k}\left(\mathcal{K}_{\beta}^{+}(t)\right)$ if $j \geq k+1$ and proves the claim.

By the claim it follows that $\left|\mathcal{B}_{k}\left(\mathcal{K}_{\beta}^{0}(t)\right)\right| \leq(k+1)\left|\mathcal{B}_{k}\left(\mathcal{K}_{\beta}^{+}(t)\right)\right|$. Using that $\mathcal{K}_{\beta}(t)=$ $\mathcal{K}_{\beta}^{0}(t) \cup \mathcal{K}_{\beta}^{+}(t)$ we have

$$
\left|\mathcal{B}_{k}\left(\mathcal{K}_{\beta}(t)\right)\right| \leq(k+2)\left|\mathcal{B}_{k}\left(\mathcal{K}_{\beta}^{+}(t)\right)\right| \text { for all } k \geq 1 .
$$

Taking logarithms, dividing both sides by $k$ and letting $k \rightarrow \infty$, we conclude that $h_{\text {top }}\left(\mathcal{K}_{\beta}(t)\right) \leq h_{\text {top }}\left(\mathcal{K}_{\beta}^{+}(t)\right)$, which gives the result.

2.4. The size of $E_{\beta}$. The results from the previous sections are enough to prove Theorem A. We start by proving the following result, which holds for all $\beta \in(1,2)$. It covers item (i) from Theorem A as well as part of Theorem B.

Proposition 2.7. For any $\beta \in(1,2)$ the bifurcation set $E_{\beta}$ is a Lebesgue null set. Furthermore, $\operatorname{dim}_{H}\left(E_{\beta} \cap[0, \delta]\right)=1$ for any $\delta>0$. In particular, $\operatorname{dim}_{H} E_{\beta}=1$.

Proof. For the first part of the statement, let $\beta \in(1,2)$ and $N \in \mathbb{N}$. The ergodicity of $T_{\beta}$ with respect to its invariant measure equivalent to the Lebesgue measure $\lambda$ implies that $\lambda$-almost every $x \in[0,1)$ is eventually mapped into the interval $(0,(1 / N))$. Hence, the survivor set $K_{\beta}((1 / N))$ is a Lebesgue null set for each $N \in \mathbb{N}$. This implies that $\lambda\left(E_{\beta}\right)=0$ since by Proposition 2.3,

$$
E_{\beta} \subseteq \bigcup_{N=1}^{\infty} K_{\beta}\left(\frac{1}{N}\right) .
$$

To prove the second part, take a large integer $N \geq 1$. Let $E_{\beta, N}$ be the set of $x \in$ $[0,1)$ with a greedy expansion $b(x, \beta)=\left(b_{i}(x, \beta)\right)$ satisfying $b_{1}(x, \beta) \ldots b_{N}(x, \beta)=$ $0^{N}$ and such that the tails $b_{N+1}(x, \beta) b_{N+2}(x, \beta) \ldots$ do not contain $N$ consecutive zeros. 
It immediately follows that $E_{\beta, N} \subseteq E_{\beta}$. Note that $K_{\beta}^{+}\left(1 / \beta^{N}\right)$ is exactly the set of $x \in[0,1)$ for which $b(x, \beta)$ does not have more than $N$ consecutive zeros. Hence,

$$
E_{\beta, N}=\frac{1}{\beta^{N}} K_{\beta}^{+}\left(\frac{1}{\beta^{N}}\right)
$$

and thus $\operatorname{dim}_{H} E_{\beta, N}=\operatorname{dim}_{H} K_{\beta}^{+}\left(1 / \beta^{N}\right)=\operatorname{dim}_{H} K_{\beta}\left(1 / \beta^{N}\right)$. Moreover, for any $\delta>0$, we can find a large integer $N$ such that $E_{\beta, n} \subseteq E_{\beta} \cap[0, \delta]$ for all $n \geq N$. Therefore,

$$
\operatorname{dim}_{H}\left(E_{\beta} \cap[0, \delta]\right) \geq \operatorname{dim}_{H} E_{\beta, n}=\operatorname{dim}_{H} K_{\beta}\left(\frac{1}{\beta^{n}}\right)
$$

for all $n \geq N$. By continuity of the map $\eta_{\beta}: t \mapsto \operatorname{dim}_{H} K_{\beta}(t)$, letting $n \rightarrow \infty$ gives that

$$
\operatorname{dim}_{H}\left(E_{\beta} \cap[0, \delta]\right) \geq \operatorname{dim}_{H} K_{\beta}(0)=\operatorname{dim}_{H}[0,1)=1 .
$$

Proof of Theorem A. Item (i) is given by Proposition 2.7. For item (ii), first bullet point, the fact that $\eta_{\beta}$ decreases weakly immediately follows from its definition and the continuity of $\eta_{\beta}$ is given by Proposition 2.5. For the second bullet point we have that the set-valued map $t \mapsto K_{\beta}(t)$ is locally constant Lebesgue almost everywhere since $\lambda\left(E_{\beta}\right)=0$. The last bullet point follows since $\eta_{\beta}(0)=1$ and for $t \geq(1 / \beta)$ we completely remove the second branch from $T_{\beta}$, so that obviously $\operatorname{dim}_{H}\left(K_{\beta}(t)\right)=0$ and $\eta_{\beta}(t)=0$.

\section{Topological structure of $E_{\beta}$}

In this section we prove Theorem $\mathrm{B}$. In fact, we prove a stronger result by specifying the set of $\beta \in(1,2)$ for which there is a $\delta>0$ such that $E_{\beta}^{+} \cap[0, \delta]$ does not contain isolated points. This is the set

$$
C_{3}:=\{\beta \in(1,2) \text { : the length of consecutive zeros in } \alpha(\beta) \text { is bounded }\} .
$$

From a dynamical point of view $C_{3}$ is the set of $\beta \in(1,2)$ such that the orbit $\left\{\widetilde{T}_{\beta}^{n}(1)\right\}_{n=0}^{\infty}$ is bounded away from zero. Replacing $\alpha(\beta)$ in the definition of $C_{3}$ by $b(1, \beta)$ gives the set called $C_{3}$ in [Sch97]. In [Sch97] Schmeling proved that this set has zero Lebesgue measure and full Hausdorff dimension. Since the two versions of $C_{3}$ only differ by countably many points, the same holds for our set $C_{3}$ from (3.1). We prove Theorem B using Lyndon words, which we will define next.

Recall from (2.5) that

$$
\mathcal{E}_{\beta}^{+}=\left\{\left(t_{i}\right) \in\{0,1\}^{\mathbb{N}}:\left(t_{i}\right) \preccurlyeq \sigma^{n}\left(\left(t_{i}\right)\right) \prec \alpha(\beta) \text { for all } n \geq 0\right\} .
$$

In other words, any sequence in $\mathcal{E}_{\beta}^{+}$is the lexicographically smallest sequence in $\Sigma_{\beta}$ under the shift map $\sigma$. For this reason we recall the following definition (cf. [Lot02]).

Definition 3.1. A word $\mathbf{s}$ is called Lyndon if $\mathbf{s}$ is aperiodic and $\sigma^{n}\left(\mathbf{s}^{\infty}\right) \succcurlyeq \mathbf{s}^{\infty}$ for all $n \geq 0$.

The following lemma lists some useful properties of Lyndon words. The first and third items easily follow from the definition and we omit their proofs.

LEMMA 3.2.

(i) $s_{1} \ldots s_{m}$ is a Lyndon word if and only if

$$
s_{i+1} \ldots s_{m} \succ s_{1} \ldots s_{m-i} \quad \text { for all } 0<i<m .
$$


(ii) If $s_{1} \ldots s_{m}$ is a Lyndon word, then for any $1 \leq n<m$ with $s_{n}=0$ the word $s_{1} \ldots s_{n}^{+}$ is also Lyndon.

(iii) If $v, w$ are Lyndon words and $v w \prec w v$, then for all $n \in \mathbb{N}$ we have that $v^{n} w$ is a Lyndon word.

Proof. To prove (ii), suppose that $s_{n}=0$ for some $1 \leq n<m$. Since 1 is a Lyndon word, the statement holds for $n=1$. If $2 \leq n<m$, then by (i) it follows that

$$
s_{i+1} \ldots s_{n}^{+} \succ s_{i+1} \ldots s_{n} \succcurlyeq s_{1} \ldots s_{n-i} \text { for all } 0<i<n .
$$

Therefore, again by (i) $s_{1} \ldots s_{n}^{+}$is a Lyndon word, as required.

By taking $i=m-1$ in Lemma 3.2(i) it follows that $s_{1}=0$ and $s_{m}=1$. So, any Lyndon word of length at least two starts with 0 and ends with 1 . We use Lemma 3.2 to show that any isolated point in $E_{\beta}^{+}$has a periodic greedy $\beta$-expansion.

Proposition 3.3. Let $\beta \in(1,2]$. If $t$ is an isolated point of $E_{\beta}^{+}$, then its greedy $\beta$ expansion $b(t, \beta)$ is periodic. Moreover, no element from $E_{\beta}^{+}$is isolated in $E_{\beta}$.

The proof of this proposition is based on the following two lemmas. Together they say that any point in $E_{\beta}^{+}$with aperiodic $\beta$-expansion can be approximated from below by a sequence of points in $E_{\beta}^{+}$that have a periodic orbit under $T_{\beta}$.

Lemma 3.4. Let $\left(t_{i}\right) \in \mathcal{E}_{\beta}^{+}$be an aperiodic sequence. For each $m \geq 1$ we have

$$
\left(t_{1} \ldots t_{m}\right)^{\infty} \prec\left(t_{i}\right) \text { and }\left(t_{1} \ldots t_{m}\right)^{\infty} \in \Sigma_{\beta} .
$$

Proof. Let $\left(t_{i}\right) \in \mathcal{E}_{\beta}^{+}$be an aperiodic sequence. Then by (2.5) we have

$$
\left(t_{i}\right) \prec \sigma^{n}\left(\left(t_{i}\right)\right) \prec \alpha(\beta) \text { for all } n \geq 1 .
$$

Fix $m \geq 1$. By taking $n=m, 2 m, \ldots$ in (3.2) it follows that

$$
\begin{aligned}
\left(t_{1} \ldots t_{m}\right)^{\infty} & =t_{1} \ldots t_{m}\left(t_{1} \ldots t_{m}\right)^{\infty} \\
& \preccurlyeq t_{1} \ldots t_{m} t_{m+1} \ldots t_{2 m}\left(t_{1} \ldots t_{m}\right)^{\infty} \\
& \preccurlyeq t_{1} \ldots t_{2 m} t_{2 m+1} \ldots t_{3 m}\left(t_{1} \ldots t_{m}\right)^{\infty} \preccurlyeq \ldots \preccurlyeq\left(t_{i}\right) .
\end{aligned}
$$

Since $\left(t_{i}\right)$ is not periodic, we conclude that $\left(t_{1} \ldots t_{m}\right)^{\infty} \prec\left(t_{i}\right)$.

For the second statement, (3.2) and the first part of the proposition give that

$$
\sigma^{n}\left(\left(t_{1} \ldots t_{m}\right)^{\infty}\right)=t_{n+1} \ldots t_{m}\left(t_{1} \ldots t_{m}\right)^{\infty} \prec t_{n+1} \ldots t_{m} t_{m+1} t_{m+2} \ldots \prec \alpha(\beta)
$$

for each $0 \leq n<m$ and hence $\left(t_{1} \ldots t_{m}\right)^{\infty} \in \Sigma_{\beta}$.

From [SM94, Proposition 2.2] we have the following lemma.

LEMMA 3.5. Let $\left(t_{i}\right) \in \mathcal{E}_{\beta}^{+}$be an aperiodic sequence. Then there exist infinitely many $m \in \mathbb{N}$ such that $t_{1} \ldots t_{m}$ is a Lyndon word.

Note that both previous lemmas do not hold for $\mathcal{E}_{\beta}$. Let $\left(t_{i}\right) \in \mathcal{E}_{\beta}^{0}$ be such that $\sigma^{n}\left(\left(t_{i}\right)\right)$ $=0^{\infty}$. Then for any $m>n$ we have $\left(t_{1} \ldots t_{m}\right)^{\infty} \succ\left(t_{i}\right)$, contradicting the statement of Lemma 3.4. As for the statement of Lemma 3.5, for all $m \geq 2 n$ we have that $t_{1} \ldots t_{m}$ is not Lyndon. 
Proof of Proposition 3.3. Let $t \in E_{\beta}^{+}$be a point with aperiodic greedy $\beta$-expansion $b(t, \beta)=\left(t_{i}\right)$. Since $\left(t_{i}\right) \in \mathcal{E}_{\beta}^{+}$, by Lemma 3.5 there exists a sequence $\left(m_{j}\right)$ such that $t_{1} \ldots t_{m_{j}}$ is Lyndon for all $j \geq 1$. Furthermore, by Lemma 3.4 we have $\left(t_{1} \ldots t_{m_{j}}\right)^{\infty} \in \Sigma_{\beta}$ for each $j \geq 1$. Hence, for all $j \geq 1$ we have $\left(t_{1} \ldots t_{m_{j}}\right)^{\infty} \in \mathcal{E}_{\beta}^{+}$and thus $\pi_{\beta}\left(\left(t_{1} \ldots t_{m_{j}}\right)^{\infty}\right) \in E_{\beta}^{+}$. Letting $j \rightarrow \infty$ we conclude that $\pi_{\beta}\left(\left(t_{1} \ldots t_{m_{j}}\right)^{\infty}\right) \rightarrow$ $\pi_{\beta}\left(\left(t_{i}\right)\right)=t$, which implies that $t$ is not isolated in $E_{\beta}^{+}$.

Now assume that $t \in E_{\beta}^{+}$has a periodic greedy $\beta$-expansion $b(t, \beta)=\left(t_{1} \ldots t_{m}\right)^{\infty}$, where $m$ is chosen minimal. We will show that $t$ is not isolated in $E_{\beta}$. If $m=1$, then we have $b(t, \beta)=0^{\infty}$, i.e., $t=0$. In this case the result trivially follows from Proposition 2.7. Now assume that $m \geq 2$. Let $a_{1} \ldots a_{m}$ be the maximal cyclic permutation of $t_{1} \ldots t_{m}$. Then there exists a $j \in\{0,1, \ldots, m-1\}$ such that $a_{1} \ldots a_{m}=t_{j+1} \ldots t_{m} t_{1} \ldots t_{j}$. Note that $\sigma^{n}\left(\left(t_{1} \ldots t_{m}\right)^{\infty}\right) \prec \alpha(\beta)$ for all $n \geq 0$. Then

$$
\left(a_{1} \ldots a_{m}\right)^{\infty} \prec \alpha(\beta),
$$

which implies that $a_{1} \ldots a_{m} \preccurlyeq \alpha_{1}(\beta) \ldots \alpha_{m}(\beta)$. We claim that $a_{1} \ldots a_{m} \prec$ $\alpha_{1}(\beta) \ldots \alpha_{m}(\beta)$.

If $a_{1} \ldots a_{m}=\alpha_{1}(\beta) \ldots \alpha_{m}(\beta)$, then (3.3) together with Lemma 2.1 gives

$$
a_{1} \ldots a_{m} \preccurlyeq \alpha_{m+1}(\beta) \ldots \alpha_{2 m}(\beta) \preccurlyeq \alpha_{1}(\beta) \ldots \alpha_{m}(\beta)=a_{1} \ldots a_{m} .
$$

So, $a_{1} \ldots a_{2 m}=\left(a_{1} \ldots a_{m}\right)^{2}$. Iterating this argument with Lemma 2.1 and (3.3) gives that $\alpha(\beta)=\left(a_{1} \ldots a_{m}\right)^{\infty}$, leading to a contradiction with (3.3). This proves the claim.

For $N \in \mathbb{N}$ define the sequence $\mathbf{t}_{N}:=\left(t_{1} \ldots t_{m}\right)^{N} t_{1} \ldots t_{j}^{+} 0^{\infty}$. Since $t_{j}=0$, the sequence $\mathbf{t}_{N}$ is well defined. By Lemma 3.2(iii) it follows that $\sigma^{n}\left(\mathbf{t}_{N}\right) \succ \mathbf{t}_{N}$ for all $0 \leq n<m N+j$. Moreover, $a_{1} \ldots a_{m} \prec \alpha_{1}(\beta) \ldots \alpha_{m}(\beta)$. It follows that $\sigma^{n}\left(\mathbf{t}_{N}\right) \prec \alpha(\beta)$ for all $n \geq 0$. So, $\mathbf{t}_{N} \in \mathcal{E}_{\beta}^{0}$ for all $N \in \mathbb{N}$. Since $\pi_{\beta}\left(\mathbf{t}_{N}\right) \searrow t$ as $N \rightarrow \infty$, the point $t \in E_{\beta}^{+}$ is not isolated in $E_{\beta}$.

The next proposition says that no point from $E_{\beta}^{0} \backslash\{0\}$ can be approximated from above by elements from $E_{\beta}$ and that a point $t \in E_{\beta}^{0} \backslash\{0\}$ is isolated in $E_{\beta}$ if the orbit of 1 enters $(0, t)$

Proposition 3.6. Let $t \in E_{\beta}^{0} \backslash\{0\}$. Then there is a $\delta>0$ such that $E_{\beta} \cap[t, t+\delta]=\{t\}$. Moreover, if $\beta-1 \notin K_{\beta}(t)$, then $t$ is isolated in $E_{\beta}$.

Proof. If $t \in E_{\beta}^{0} \backslash\{0\}$, then there is a smallest $n \geq 0$ such that $T_{\beta}^{n}(t)=(1 / \beta)$. By the rightcontinuity of $T_{\beta}$, there is a $\delta>0$ such that all $\epsilon \in(t, t+\delta]$ satisfy $T_{\beta}^{n+1}(\epsilon) \in(0, t) \subseteq$ $(0, \epsilon)$. Hence, $\epsilon \notin K_{\beta}(\epsilon)$ and thus $\epsilon \notin E_{\beta}$.

The first statement implies that to prove that an element from $E_{\beta}^{0} \backslash\{0\}$ is isolated, it is enough to prove that it cannot be approximated from below. If again $n$ is such that $T_{\beta}^{n}(t)=(1 / \beta)$, then for a small enough $\delta$ we know that for any point $\epsilon \in[t-\delta, t)$ the point $T_{\beta}^{n+1}(\epsilon)$ is close to 1 . Let $m$ be the smallest integer such that $T_{\beta}^{m}(\beta-1) \in(0, t)$. Then there is a $0<\delta<t-T_{\beta}^{m}(\beta-1)$ such that any $\epsilon \in[t-\delta, t)$ satisfies

$$
T_{\beta}^{n+1+m+1}(\epsilon) \in\left(0, T_{\beta}^{m}(\beta-1)\right) \subseteq(0, \epsilon) .
$$

Hence, $\epsilon \notin E_{\beta}$ and $E_{\beta} \cap[t-\delta, t]=\{t\}$. 
From now on we focus on the set $E_{\beta}^{+}$. We first construct subintervals of $(1,2)$ such that $E_{\beta}^{+}$contains isolated points whenever $\beta$ is in one of these intervals. We start with a couple of lemmas.

Lemma 3.7. Let $\left(t_{i}\right),\left(\alpha_{i}\right) \in\{0,1\}^{\mathbb{N}}$ be given. Suppose that there is an $m \geq 1$ such that $\alpha_{m}=1$ and $\sigma^{m}\left(\left(\alpha_{i}\right)\right) \preccurlyeq\left(t_{i}\right)$. Define the sets

$$
\begin{aligned}
\mathcal{K} & :=\left\{\left(x_{i}\right) \in\{0,1\}^{\mathbb{N}}:\left(t_{i}\right) \preccurlyeq \sigma^{n}\left(\left(x_{i}\right)\right) \prec\left(\alpha_{i}\right) \text { for all } n \geq 0\right\}, \\
\mathcal{X}_{m} & :=\left\{\left(x_{i}\right) \in\{0,1\}^{\mathbb{N}}:\left(t_{i}\right) \preccurlyeq \sigma^{n}\left(\left(x_{i}\right)\right) \preccurlyeq\left(\alpha_{1} \ldots \alpha_{m}^{-}\right)^{\infty} \text { for all } n \geq 0\right\} .
\end{aligned}
$$

Then $\mathcal{K}=\mathcal{X}_{m}$.

Proof. Obviously, $\mathcal{X}_{m} \subseteq \mathcal{K}$. We show that $\mathcal{K} \backslash \mathcal{X}_{m}=\emptyset$. Suppose that this is not the case and let $\left(x_{i}\right) \in \mathcal{K} \backslash \mathcal{X}_{m}$. Then there is a $j \geq 1$ such that $x_{j+1} \ldots x_{j+m}=\alpha_{1} \ldots \alpha_{m}$. Since $\left(x_{i}\right) \in \mathcal{K}$, the assumption that $\sigma^{m}\left(\left(\alpha_{i}\right)\right) \preccurlyeq\left(t_{i}\right)$ implies that

$$
x_{j+m+1} x_{j+m+2} \ldots \prec \alpha_{m+1} \alpha_{m+2} \ldots \preccurlyeq\left(t_{i}\right),
$$

which contradicts $\left(x_{i}\right) \in \mathcal{K}$. Hence, $\mathcal{K} \backslash \mathcal{X}_{m}=\emptyset$.

Let $\beta \in(1,2)$ and $t \in[0,1)$. The previous lemma has the following consequence for $\mathcal{K}_{\beta}^{+}(t)$. If there is a smallest $m \geq 1$ such that

$$
\alpha_{m+1}(\beta) \alpha_{m+2}(\beta) \ldots \preccurlyeq b(t, \beta)
$$

or equivalently $\tilde{T}_{\beta}^{m}(1) \leq t$, then we can rewrite $\mathcal{K}_{\beta}^{+}(t)$ as

$$
\mathcal{K}_{\beta}^{+}(t)=\left\{\left(x_{i}\right): b(t, \beta) \preccurlyeq \sigma^{n}\left(\left(x_{i}\right)\right) \preccurlyeq\left(\alpha_{1}(\beta) \ldots \alpha_{m}(\beta)^{-}\right)^{\infty} \text { for any } n \geq 0\right\} .
$$

Hence, any point in the survivor set $K_{\beta}^{+}(t)$ then has the property that its entire orbit lies between $t$ and the point $\pi_{\beta}\left(\left(\alpha_{1}(\beta) \ldots \alpha_{m}(\beta)^{-}\right)^{\infty}\right)$. We need two more lemmas. Recall the definition of the set $\mathcal{Q}$ from (2.2) as the set of sequences that occur as $\alpha(\beta)$ for some $\beta \in(1,2]$.

LEMMA 3.8. Let $\left(a_{1} \ldots a_{m}\right)^{\infty} \in \mathcal{Q}$ with minimal period $m$. Then

$$
a_{i+1} \ldots a_{m}^{+} \preccurlyeq a_{1} \ldots a_{m-i} \quad \text { for all } 0<i<m .
$$

Proof. Let $\beta \in(1,2)$ be such that $\alpha(\beta)=\left(a_{1} \ldots a_{m}\right)^{\infty}$. Then $b(1, \beta)=a_{1} \ldots a_{m}^{+} 0^{\infty}$. Hence, for each $0<i<m$ we have $b\left(T_{\beta}^{i}(1), \beta\right)=a_{i+1} \ldots a_{m}^{+} 0^{\infty}$ and $T_{\beta}^{i}(1)<1$. The result then follows from Lemma 2.2.

Note that for any non-periodic word $b_{1} \ldots b_{m} \in\{0,1\}^{*}$ there is a $0 \leq j \leq m-1$ such that $b_{j+1} \ldots b_{m} b_{1} \ldots b_{j}$ is the smallest among its cyclic permutations and therefore Lyndon. We denote this word by $\mathbf{S}\left(b_{1} \ldots b_{m}\right)$ and call it the Lyndon word for $b_{1} \ldots b_{m}$. Similarly, there is a $0 \leq k \leq m-1$ such that $b_{k+1} \ldots b_{m} b_{1} \ldots b_{k}$ is the largest among its cyclic permutations. We denote this by word by $\mathbf{L}\left(b_{1} \ldots b_{m}\right)$. In what follows we will sometimes use the property that for any word $b_{1} \ldots b_{m} \in\{0,1\}^{m}$ and any sequence $\left(x_{i}\right) \in\{0,1\}^{\mathbb{N}}$,

$$
\sigma^{n}\left(\left(x_{i}\right)\right) \succcurlyeq b_{1} \ldots b_{m} 0^{\infty} \text { for all } n \geq 0 \quad \Longleftrightarrow \quad \sigma^{n}\left(\left(x_{i}\right)\right) \succcurlyeq\left(b_{1} \ldots b_{m}\right)^{\infty} \text { for all } n \geq 0 \text {. }
$$




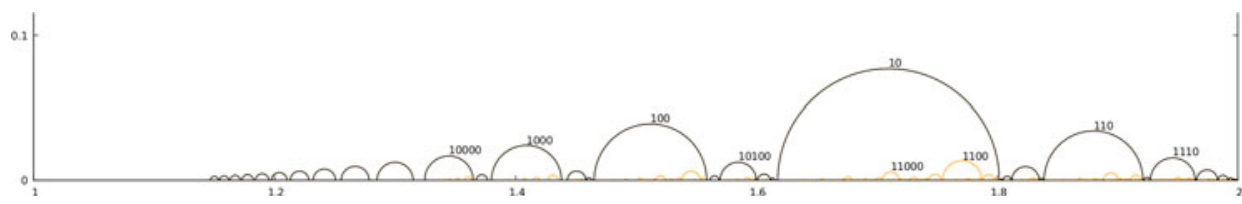

FIGURE 2. Some of the basic intervals $\left(\beta_{L}, \beta_{R}\right.$ ]. The numbers near the arches indicate the words $a_{1} \ldots a_{m}$ such that $\alpha\left(\beta_{L}\right)=\left(a_{1} \ldots a_{m}\right)^{\infty}$. The intervals that are not contained in any other interval are the Farey intervals. They are the ones for which $a_{1} \ldots a_{m}$ is a Farey word. The arches corresponding to Farey intervals are shown in black, the lighter coloured arches correspond to words that are Lyndon, but not Farey.

LEMMA 3.9. Let $s_{1} \ldots s_{m}$ be a Lyndon word and write $a_{1} \ldots a_{m}=\mathbf{L}\left(s_{1} \ldots s_{m}\right)$. Let $0 \leq j<m$ be such that $s_{1} \ldots s_{m}=a_{j+1} \ldots a_{m} a_{1} \ldots a_{j}$ and set

$$
\mathcal{Z}_{m}:=\left\{\left(x_{i}\right) \in\{0,1\}^{\mathbb{N}}: s_{1} \ldots s_{m} 0^{\infty} \preccurlyeq \sigma^{n}\left(\left(x_{i}\right)\right) \preccurlyeq\left(a_{1} \ldots a_{m}\right)^{\infty} \forall n \geq 0\right\} .
$$

(i) If $\left(x_{i}\right) \in \mathcal{Z}_{m}$ has prefix $a_{j+1} \ldots a_{m}$, then $\left(x_{i}\right)=\left(s_{1} \ldots s_{m}\right)^{\infty}$.

(ii) If $\left(x_{i}\right) \in \mathcal{Z}_{m}$ has prefix $a_{1} \ldots a_{j}$, then $\left(x_{i}\right)=\left(a_{1} \ldots a_{m}\right)^{\infty}$.

Proof. Since the proofs of (i) and (ii) are similar, we only give the proof of (i). Let $a_{j+1} \ldots a_{m} x_{1} x_{2} \ldots \in \mathcal{Z}_{m}$. Then

$$
s_{1} \ldots s_{m} 0^{\infty} \preccurlyeq \sigma^{n}\left(a_{j+1} \ldots a_{m} x_{1} x_{2} \ldots\right) \preccurlyeq\left(a_{1} \ldots a_{m}\right)^{\infty} \text { for all } n \geq 0 .
$$

In particular,

$$
a_{j+1} \ldots a_{m} x_{1} \ldots x_{j} \succcurlyeq s_{1} \ldots s_{m}=a_{j+1} \ldots a_{m} a_{1} \ldots a_{j}
$$

which gives

$$
x_{1} \ldots x_{j} \succcurlyeq a_{1} \ldots a_{j}
$$

On the other hand, by taking $n=m-j$ in (3.5) we get $x_{1} \ldots x_{m} \preccurlyeq a_{1} \ldots a_{m}$. Hence,

$$
x_{1} \ldots x_{j}=a_{1} \ldots a_{j} \quad \text { and } \quad x_{j+1} \ldots x_{m} \preccurlyeq a_{j+1} \ldots a_{m} .
$$

Again, by (3.5) now with $n=m$ we have $x_{j+1} \ldots x_{m} \succcurlyeq s_{1} \ldots s_{m-j}=a_{j+1} \ldots a_{m}$. Therefore, $x_{1} \ldots x_{m}=a_{1} \ldots a_{m}$. By iteration we conclude that

$$
a_{j+1} \ldots a_{m} x_{1} x_{2} \ldots=\left(a_{j+1} \ldots a_{m} a_{1} \ldots a_{j}\right)^{\infty}=\left(s_{1} \ldots s_{m}\right)^{\infty},
$$

as required.

We now construct infinitely many nested intervals $\left(\beta_{L}, \beta_{R}\right]$ such that $E_{\beta}^{+}$has isolated points whenever $\beta \in\left(\beta_{L}, \beta_{R}\right]$. Figure 2 shows some of these intervals. We will later show that these basic intervals cover the whole interval $(1,2)$ up to a set of zero Lebesgue measure.

Proposition 3.10. Let $s_{1} \ldots s_{m}$ be a Lyndon word and write $a_{1} \ldots a_{m}=\mathbf{L}\left(s_{1} \ldots s_{m}\right)$. Then both $\left(a_{1} \ldots a_{m}\right)^{\infty}$ and $a_{1} \ldots a_{m}^{+}\left(s_{1} \ldots s_{m}\right)^{\infty}$ belong to $\mathcal{Q}$ and hence there are uniquely defined bases $\beta_{L}, \beta_{R} \in(1,2]$ such that $\alpha\left(\beta_{L}\right)=\left(a_{1} \ldots a_{m}\right)^{\infty}$ and $\alpha\left(\beta_{R}\right)=$ $a_{1} \ldots a_{m}^{+}\left(s_{1} \ldots s_{m}\right)^{\infty}$. Moreover:

(i) $\left(s_{1} \ldots s_{m}\right)^{\infty} \in \Sigma_{\beta}$ if and only if $\beta>\beta_{L}$;

(ii) if $\beta \in\left(\beta_{L}, \beta_{R}\right]$, then $\pi_{\beta}\left(\left(s_{1} \ldots s_{m}\right)^{\infty}\right)$ is an isolated point of $E_{\beta}^{+}$;

(iii) if $\beta>\beta_{R}$, then $\pi_{\beta}\left(\left(s_{1} \ldots s_{m}\right)^{\infty}\right)$ is not an isolated point of $E_{\beta}^{+}$. 
Proof. Let $\beta_{L}$ be as in the proposition. First we show that the interval $\left(\beta_{L}, \beta_{R}\right]$ is well defined, i.e., $\beta_{R}$ exists and that $\beta_{L}<\beta_{R}$. We use the characterization from Lemma 2.1, so it suffices to show that the sequence $\mathbf{a}=a_{1} \ldots a_{m}^{+}\left(s_{1} \ldots s_{m}\right)^{\infty} \in \mathcal{Q}$, i.e., it satisfies $\sigma^{n}(\mathbf{a}) \preccurlyeq \mathbf{a}$ for all $n \geq 0$. Since $s_{1} \ldots s_{m}$ is a Lyndon word, any word of length $1 \leq n \leq$ $m-1$ occurring in $a_{1} \ldots a_{m}=\mathbf{L}\left(s_{1} \ldots s_{m}\right)$ is lexicographically larger than or equal to $s_{1} \ldots s_{n}$. Combining this with Lemmas 3.8 and 3.2(i) gives

$$
a_{n+1} \ldots a_{m}^{+} s_{1} \ldots s_{n} \preccurlyeq a_{1} \ldots a_{m-n} a_{m-n+1} \ldots a_{m} \prec a_{1} \ldots a_{m}^{+}
$$

for all $0<n<m$. So, $\sigma^{n}(\mathbf{a}) \prec \mathbf{a}$ for each $0<n<m$. Moreover, since

$$
\sigma^{n}\left(\left(s_{1} \ldots s_{m}\right)^{\infty}\right) \preccurlyeq\left(a_{1} \ldots a_{m}\right)^{\infty} \prec a_{1} \ldots a_{m}^{+}\left(s_{1} \ldots s_{m}\right)^{\infty}
$$

for all $n \geq 0$, we get $\sigma^{n}(\mathbf{a}) \prec \mathbf{a}$ for all $n \geq 1$ and thus $\mathbf{a} \in \mathcal{Q}$. Lemma 2.1 then implies that $\mathbf{a}$ is indeed the quasi-greedy expansion of 1 for some base $\beta_{R}$, i.e., $\alpha\left(\beta_{R}\right)=$ $a_{1} \ldots a_{m}^{+}\left(s_{1} \ldots s_{m}\right)^{\infty}$. Since $\alpha\left(\beta_{L}\right) \prec \alpha\left(\beta_{R}\right)$, Lemma 2.1 also gives that $\beta_{R}>\beta_{L}$. Hence, the interval $\left(\beta_{L}, \beta_{R}\right]$ is well defined.

Let $1 \leq j \leq m-1$ be such that

$$
s_{1} \ldots s_{m}=a_{j+1} \ldots a_{m} a_{1} \ldots a_{j} .
$$

For (i), note that if $\beta \leq \beta_{L}$, then $\left(s_{1} \ldots s_{m}\right)^{\infty} \notin \Sigma_{\beta}$ since

$$
\sigma^{j}\left(\left(s_{1} \ldots s_{m}\right)^{\infty}\right)=\left(a_{1} \ldots a_{m}\right)^{\infty} \succcurlyeq \alpha(\beta) .
$$

For $\beta \in\left(\beta_{L}, \beta_{R}\right]$ it follows immediately that $\left(s_{1} \ldots s_{m}\right)^{\infty} \in \Sigma_{\beta}$ since $s_{1} \ldots s_{m}$ is the smallest permutation of $a_{1} \ldots a_{m}$ and $\left(a_{1} \ldots a_{m}\right)^{\infty} \prec \alpha(\beta)$.

For (ii), let $\beta \in\left(\beta_{L}, \beta_{R}\right]$ and set $t=\pi_{\beta}\left(\left(s_{1} \ldots s_{m}\right)^{\infty}\right)$. Then $b(t, \beta)=\left(s_{1} \ldots s_{m}\right)^{\infty} \in$ $\mathcal{E}_{\beta}^{+}$, so $t \in E_{\beta}^{+}$. By Lemma 2.2 and since $t$ has a periodic $\beta$-expansion, there exists a small $\delta>0$ such that for any $x \in[t-\delta, t+\delta]$ the greedy expansion $b(x, \beta)$ has prefix $s_{1} \ldots s_{m}$. By Lemma 3.7 it follows that

$$
\begin{aligned}
\mathcal{K}_{\beta}^{+}(t-\delta) & \subseteq\left\{\left(x_{i}\right): s_{1} \ldots s_{m} 0^{\infty} \preccurlyeq \sigma^{n}\left(\left(x_{i}\right)\right) \prec a_{1} \ldots a_{m}^{+}\left(s_{1} \ldots s_{m}\right)^{\infty} \forall n \geq 0\right\} \\
& =\left\{\left(x_{i}\right):\left(s_{1} \ldots s_{m}\right)^{\infty} \preccurlyeq \sigma^{n}\left(\left(x_{i}\right)\right) \prec a_{1} \ldots a_{m}^{+}\left(s_{1} \ldots s_{m}\right)^{\infty} \forall n \geq 0\right\} \\
& =\left\{\left(x_{i}\right):\left(s_{1} \ldots s_{m}\right)^{\infty} \preccurlyeq \sigma^{n}\left(\left(x_{i}\right)\right) \preccurlyeq\left(a_{1} \ldots a_{m}\right)^{\infty} \forall n \geq 0\right\} \\
& =\left\{\left(x_{i}\right): s_{1} \ldots s_{m} 0^{\infty} \preccurlyeq \sigma^{n}\left(\left(x_{i}\right)\right) \preccurlyeq\left(a_{1} \ldots a_{m}\right)^{\infty} \forall n \geq 0\right\},
\end{aligned}
$$

where we have used the fact from (3.4) in the first and last equalities. Since for any $x \in[t-\delta, t+\delta]$ the greedy expansion $b(x, \beta)$ begins with $s_{1} \ldots s_{m}$, by Lemma 3.9(i) and (3.6) we obtain that

$$
K_{\beta}^{+}(t-\delta) \cap[t-\delta, t+\delta] \subseteq\{t\}
$$

Since $t \in E_{\beta}^{+} \cap[t-\delta, t+\delta] \subseteq K_{\beta}^{+}(t-\delta) \cap[t-\delta, t+\delta]$, we conclude that $t$ is isolated in $E_{\beta}^{+}$for any $\beta \in\left(\beta_{L}, \beta_{R}\right]$.

For (iii), let $\beta>\beta_{R}$ and again set $t=\pi_{\beta}\left(\left(s_{1} \ldots s_{m}\right)^{\infty}\right)$. We construct a sequence $\left(\mathbf{t}_{n}\right)$ in $\mathcal{E}_{\beta}^{+}$such that $\mathbf{t}_{n} \searrow\left(s_{1} \ldots s_{m}\right)^{\infty}$ in the order topology as $n \rightarrow \infty$. Let

$$
\mathbf{t}_{n}:=\left(\left(s_{1} \ldots s_{m}\right)^{n} s_{1} \ldots s_{m-j}^{+}\right)^{\infty}=\left(\left(a_{j+1} \ldots a_{m} a_{1} \ldots a_{j}\right)^{n} a_{j+1} \ldots a_{m}^{+}\right)^{\infty} .
$$


We claim that there is an $N \in \mathbb{N}$ such that $\mathbf{t}_{n} \in \mathcal{E}_{\beta}^{+}$for all $n>N$. By Lemma 3.2(ii) and (iii) it follows that $\mathbf{t}_{n}$ is Lyndon. Left to show is that $\mathbf{t}_{n} \in \Sigma_{\beta}$. Note that the largest permutation of $\mathbf{t}_{n}$ is given by

$$
\begin{aligned}
\mathbf{d}_{n} & =\left(a_{1} \ldots a_{m}^{+}\left(a_{j+1} \ldots a_{m} a_{1} \ldots a_{j}\right)^{n-1} a_{j+1} \ldots a_{m}\right)^{\infty} \\
& =\left(a_{1} \ldots a_{m}^{+}\left(s_{1} \ldots s_{m}\right)^{n-1} s_{1} \ldots s_{m-j}\right)^{\infty} .
\end{aligned}
$$

For $\beta>\beta_{R}$ either $\alpha_{1}(\beta) \ldots \alpha_{m}(\beta) \succ a_{1} \ldots a_{m}^{+}$or there exists an $N \geq 1$ such that $\alpha(\beta)=$ $a_{1} \ldots a_{m}^{+}\left(s_{1} \ldots s_{m}\right)^{N-1} b_{1} \ldots b_{m}$ with $b_{1} \ldots b_{m} \succ s_{1} \ldots s_{m}$. In the first case obviously $\mathbf{d}_{n} \prec \alpha(\beta)$. In the second case we have $\mathbf{d}_{n} \prec \alpha(\beta)$ for all $n>N$. Hence, $\mathbf{t}_{n} \in \Sigma_{\beta}$ for all $n>N$.

We have found a sequence $\left(\mathbf{t}_{n}\right) \subseteq \mathcal{E}_{\beta}^{+}$decreasing to $b(t, \beta)=\left(s_{1} \ldots s_{m}\right)^{\infty}$ as $n \rightarrow \infty$ and, accordingly, a sequence $\left(\pi_{\beta}\left(\mathbf{t}_{n}\right)\right) \subseteq E_{\beta}^{+}$decreasing to $t=\pi_{\beta}\left(\left(s_{1} \ldots s_{m}\right)^{\infty}\right)$ as $n \rightarrow \infty$. Therefore, $t$ is not isolated in $E_{\beta}^{+}$.

Recall from (3.1) that $C_{3}$ is the set of $\beta \in(1,2)$ such that the length of consecutive zeros in the quasi-greedy expansion $\alpha(\beta)$ is bounded.

THEOREM 3.11. If $\beta \in(1,2) \backslash C_{3}$, then both $E_{\beta} \cap[0, \delta]$ and $E_{\beta}^{+} \cap[0, \delta]$ contain infinitely many isolated points and infinitely many accumulation points for all $\delta>0$.

Proof. By Proposition 2.7 it follows that $E_{\beta} \cap[0, \delta]$ and $E_{\beta}^{+} \cap[0, \delta]$ contain infinitely many accumulation points for all $\delta>0$, so we focus on the isolated points. Fix $\beta \in$ $(1,2) \backslash C_{3}$. Then $\alpha(\beta)$ contains consecutive zeros of arbitrary length. Hence, $\alpha(\beta)$ is not periodic and the orbit of 1 under $\widetilde{T}_{\beta}$ will come arbitrarily close to 0 . This implies that for any $t>0, \beta-1 \notin K_{\beta}(t)$ and thus by Proposition 3.6 any $t \in E_{\beta}^{0} \backslash\{0\}$ will be isolated in $E_{\beta}$. Note that for any $n \geq 1$ we have $\left(1 / \beta^{n}\right) \in E_{\beta}^{0}$. This gives the statement for $E_{\beta}$.

To prove that $E_{\beta}^{+}$contains infinitely many isolated points arbitrarily close to 0 , we construct by induction a sequence of intervals $\left(\beta_{L, k}, \beta_{R, k}\right), k \geq 1$, such that $\beta \in$ $\left(\beta_{L, k}, \beta_{R, k}\right)$ for all $k \geq 1$, where $\left(\beta_{L, k}, \beta_{R, k}\right)$ is defined as in Proposition 3.10. Write

$$
\alpha(\beta)=1^{l_{1}} 0^{m_{1}} 1^{l_{2}} 0^{m_{2}} \ldots 1^{l_{k}} 0^{m_{k}} \ldots
$$

Since $\alpha(\beta)$ does not end with $0^{\infty}$, we have $m_{k} \in\{1,2, \ldots\}$ for all $k \geq 1$. Furthermore, from $\beta \notin C_{3}$ we get $\sup _{k \geq 1} m_{k}=\infty$.

Set $i_{0}=1$ and let $i_{1}>i_{0}$ be the smallest index for which $m_{i_{1}}>m_{1}$. Set $\mathbf{a}_{1}:=$ $1^{l_{1}} 0^{m_{1}} \cdots 1^{l_{i_{1}}-1} 0$. Note that $\alpha(\beta)$ begins with $\mathbf{a}_{1}^{+}$and by Lemma $2.1 \sigma^{n}(\alpha(\beta)) \preccurlyeq \alpha(\beta)$ for all $n \geq 0$. This implies that $\sigma^{n}\left(\mathbf{a}_{1}^{\infty}\right) \preccurlyeq \mathbf{a}_{1}^{\infty}$ for all $n \geq 0$. So, by Lemma 2.1 the sequence $\mathbf{a}_{1}^{\infty}$ is the quasi-greedy expansion of 1 for some base $\beta_{L, 1}$, i.e., $\alpha\left(\beta_{L, 1}\right)=\mathbf{a}_{1}^{\infty}$. Note that the word $\mathbf{a}_{1}$ contains consecutive zeros of length at most $m_{1}$. So, the Lyndon word $\mathbf{s}_{1}=$ $s_{1} \ldots s_{l_{1}+m_{1}+\cdots+l_{i_{1}}}$ for $\mathbf{a}_{1}$ begins with $0^{m_{1}} 1$. Again, one can check that $\sigma^{n}\left(\mathbf{a}_{1}^{+} \mathbf{s}_{1}^{\infty}\right) \preccurlyeq \mathbf{a}_{1}^{+} \mathbf{s}_{1}^{\infty}$ for all $n \geq 0$. So, there exists $\beta_{R, 1} \in(1,2)$ such that $\alpha\left(\beta_{R, 1}\right)=\mathbf{a}_{1}^{+} \mathbf{s}_{1}^{\infty}$. By using $m_{i_{1}}>m_{1}$ and (3.8) it follows that

$$
\alpha\left(\beta_{L, 1}\right)=\mathbf{a}_{1}^{\infty}=\left(1^{l_{1}} 0^{m_{1}} \cdots 1^{l_{i_{1}}-1} 0\right)^{\infty} \prec 1^{l_{1}} 0^{m_{1}} \cdots 1^{l_{i_{1}}} 0 \cdots=\alpha(\beta)
$$

and

$$
\alpha\left(\beta_{R, 1}\right)=\mathbf{a}_{1}^{+} \mathbf{s}_{1}^{\infty}=1^{l_{1}} 0^{m_{1}} \ldots 1^{l_{i_{1}}} 0^{m_{1}} 1 \cdots \succ 1^{l_{1}} 0^{m_{1}} \cdots 1^{l_{i_{1}}} 0^{m_{i_{1}}} 1 \cdots=\alpha(\beta) .
$$


By Lemma 2.1 we have $\beta \in\left(\beta_{L, 1}, \beta_{R, 1}\right)$. Moreover, by Proposition 3.10 we have that $\pi_{\beta}\left(\mathbf{s}_{1}^{\infty}\right)$ is an isolated point of $E_{\beta}^{+}$. Now we pick $i_{k}$ using $i_{k-1}$. Let $i_{k}>i_{k-1}$ be the smallest index such that $m_{i_{k}}>m_{i_{k-1}}$. Then by the definitions of $i_{1}, \ldots, i_{k-1}$ it follows that $m_{i_{k}}>m_{j}$ for all $j<i_{k}$. Set $\mathbf{a}_{k}:=1^{l_{1}} 0^{m_{1}} \ldots 1^{l_{i_{k}}-1} 0$. Then the block $\mathbf{a}_{k}$ contains consecutive zeros of length at most $m_{i_{k-1}}$. So, the Lyndon word $\mathbf{s}_{k}=s_{1} \ldots s_{l_{1}+m_{1}+\cdots+l_{i_{k}}}$ for $\mathbf{a}_{k}$ begins with $0^{m_{i}-1} 1$. By the same argument as above we can find two bases $\beta_{L, k}, \beta_{R, k} \in(1,2)$ such that

$$
\begin{aligned}
& \alpha\left(\beta_{L, k}\right)=\mathbf{a}_{k}^{\infty}=\left(1^{l_{1}} 0^{m_{1}} \cdots 1^{l_{i_{k}}-1} 0\right)^{\infty} \prec 1^{l_{1}} 0^{m_{1}} \cdots 1^{l_{i_{k}}} 0 \cdots=\alpha(\beta), \\
& \alpha\left(\beta_{R, k}\right)=\mathbf{a}_{k}^{+} \mathbf{s}_{k}^{\infty}=1^{l_{1}} 0^{m_{1}} \cdots 1^{l_{i_{k}}} 0^{m_{i_{k-1}}} 1 \cdots \succ 1^{l_{1}} 0^{m_{1}} \cdots 1^{l_{i_{k}}} 0^{m_{i_{k}}} 1 \cdots=\alpha(\beta) .
\end{aligned}
$$

Therefore, $\beta \in\left(\beta_{L, k}, \beta_{R, k}\right)$ and by Proposition 3.10 we have that $\pi_{\beta}\left(\mathbf{s}_{k}^{\infty}\right)$ is an isolated point of $E_{\beta}^{+}$.

By induction we construct a sequence of intervals $\left(\beta_{L, k}, \beta_{R, k}\right), k \geq 1$, such that $\beta \in$ $\left(\beta_{L, k}, \beta_{R, k}\right)$ for all $k \geq 1$. Moreover, the points $\pi_{\beta}\left(\mathbf{s}_{k}^{\infty}\right)$ are isolated in $E_{\beta}^{+}$. Note that $\mathbf{s}_{k}$ begins with a block $0^{m_{i k-1}} 1$ for any $k \geq 1$ and $m_{i_{k-1}}$ strictly increases to $\infty$ as $k \rightarrow \infty$. This implies that $E_{\beta}^{+} \cap[0, \delta]$ contains infinitely many isolated points for any $\delta>0$.

THEOREM 3.12. For $\beta \in C_{3}$ there is a $\delta>0$ such that $E_{\beta}^{+} \cap[0, \delta]$ has no isolated points.

Proof. Fix $\beta \in C_{3}$. Then the length of consecutive zeros in $\alpha(\beta)$ is bounded by some large integer $M$. Set $\delta=\left(1 / \beta^{M+3}\right)=\pi_{\beta}\left(0^{M+2} 10^{\infty}\right)$. To show that $E_{\beta}^{+} \cap[0, \delta]$ has no isolated points, suppose on the contrary that $t$ is an isolated point of $E_{\beta}^{+} \cap[0, \delta]$. By Proposition 3.3 it follows that the greedy $\beta$-expansion $b(t, \beta)$ of $t$ is periodic, namely

$$
b(t, \beta)=\left(t_{1} \ldots t_{m}\right)^{\infty} \in \mathcal{E}_{\beta}^{+}
$$

with minimal period $m$. Moreover, $t_{1} \ldots t_{m}$ is Lyndon. For $m=1$ we get that $t=0$, which by Proposition 2.7 is not isolated in $E_{\beta}^{+}$. Let $m \geq 2$ and let $a_{1} \ldots a_{m}=\mathbf{L}\left(t_{1} \ldots t_{m}\right)$. Then $\left(a_{1} \ldots a_{m}\right)^{\infty} \in \mathcal{Q}$, so by Lemma 2.1 it is the quasi-greedy expansion of 1 for some base $\beta_{L}$, i.e., $\alpha\left(\beta_{L}\right)=\left(a_{1} \ldots a_{m}\right)^{\infty}$. By Proposition 3.10 it follows that $\beta \in\left(\beta_{L}, \beta_{R}\right]$, where $\beta_{R}$ is the unique base satisfying

$$
\alpha\left(\beta_{R}\right)=a_{1} \ldots a_{m}^{+}\left(t_{1} \ldots t_{m}\right)^{\infty}
$$

Hence,

$$
\left(a_{1} \ldots a_{m}\right)^{\infty} \prec \alpha(\beta) \preccurlyeq a_{1} \ldots a_{m}^{+}\left(t_{1} \ldots t_{m}\right)^{\infty} .
$$

Since $t \leq \delta=\pi_{\beta}\left(0^{M+2} 10^{\infty}\right)$, we have $\left(t_{1} \ldots t_{m}\right)^{\infty}=b(t, \beta) \preccurlyeq 0^{M+2} 10^{\infty}$. So, $t_{1} \ldots t_{m}$ begins with $M+2$ consecutive zeros and $a_{1} \ldots a_{m}$ contains $M+2$ consecutive zeros. By (3.9) we conclude that $\alpha(\beta)$ contains $M+1$ consecutive zeros, leading to a contradiction with our hypothesis that the number of consecutive zeros in $\alpha(\beta)$ is bounded by $M$.

Proof of Theorem B. The first part of the statement follows from Proposition 2.7 and Theorem 3.11 since $\lambda\left(C_{3}\right)=0$ by the results from [Sch97]. The fact from [Sch97] that $\operatorname{dim}_{H} C_{3}=1$ together with Theorem 3.12 gives the last part of the result. 


\section{When $E_{\beta}^{+}$does not have isolated points}

In this section we prove Theorem $\mathrm{C}$, which states that the set of $\beta \in(1,2)$ for which $E_{\beta}^{+}$ has no isolated points is rather small; it has zero Hausdorff dimension. The theorem is obtained by showing that the intervals $\left(\beta_{L}, \beta_{R}\right]$ introduced in the previous section cover all but a Hausdorff dimension zero part of the interval $(1,2)$. Figure 2 suggests that the basic intervals are nested. In Proposition 4.1 below we prove that this is indeed the case. Subsequently, we identify those intervals $\left(\beta_{L}, \beta_{R}\right]$ that are not contained in any other basic interval, which turn out to be the ones given by a specific subset of the Lyndon words, called Farey words.

Proposition 4.1. Let $I_{1}=\left(\beta_{L}, \beta_{R}\right]$ and $I_{2}=\left(\tilde{\beta}_{L}, \tilde{\beta}_{R}\right]$ be two different basic intervals. If $I_{1} \cap I_{2} \neq \emptyset$, then $I_{1} \subset I_{2}$ or $I_{2} \subset I_{1}$.

Proof. Suppose that $I_{1}=\left(\beta_{L}, \beta_{R}\right]$ is parameterized by the word $a_{1} \ldots a_{m}$ and $I_{2}=$ $\left(\tilde{\beta}_{L}, \tilde{\beta}_{R}\right]$ is parameterized by the word $b_{1} \ldots b_{n}$, i.e.,

$$
\begin{aligned}
& \alpha\left(\beta_{L}\right)=\left(a_{1} \ldots a_{m}\right)^{\infty}, \quad \alpha\left(\beta_{R}\right)=a_{1} \ldots a_{m}^{+}\left(s_{1} \ldots s_{m}\right)^{\infty} ; \\
& \alpha\left(\tilde{\beta}_{L}\right)=\left(b_{1} \ldots b_{n}\right)^{\infty}, \quad \alpha\left(\tilde{\beta}_{R}\right)=b_{1} \ldots b_{n}^{+}\left(t_{1} \ldots t_{n}\right)^{\infty},
\end{aligned}
$$

where $s_{1} \ldots s_{m}=\mathbf{S}\left(a_{1} \ldots a_{m}\right)$ and $t_{1} \ldots t_{n}=\mathbf{S}\left(b_{1} \ldots b_{n}\right)$ are the Lyndon words for $a_{1} \ldots a_{m}$ and $b_{1} \ldots b_{n}$, respectively. Since $I_{1} \cap I_{2} \neq \emptyset$, by symmetry we may assume that $\tilde{\beta}_{L} \in I_{1}=\left(\beta_{L}, \beta_{R}\right]$. We are going to show that $\tilde{\beta}_{R}<\beta_{R}$, which by Lemma 2.1 is equivalent to showing that

$$
b_{1} \ldots b_{n}^{+}\left(t_{1} \ldots t_{n}\right)^{\infty} \prec a_{1} \ldots a_{m}^{+}\left(s_{1} \ldots s_{m}\right)^{\infty} .
$$

Since $\beta_{L}<\tilde{\beta}_{L} \leq \beta_{R}$, by Lemma 2.1 it follows that

$$
\left(a_{1} \ldots a_{m}\right)^{\infty} \prec\left(b_{1} \ldots b_{n}\right)^{\infty} \preccurlyeq a_{1} \ldots a_{m}^{+}\left(s_{1} \ldots s_{m}\right)^{\infty} .
$$

We claim that $n>m$.

- If $n<m$, then by (4.2) we have $b_{1} \ldots b_{n}=a_{1} \ldots a_{n}$. Write $m=u n+r$ with $u \geq 1$ and $1 \leq r \leq n$. By Lemma 3.8 and (4.2) it follows that $a_{1} \ldots a_{u n}=\left(b_{1} \ldots b_{n}\right)^{u}$ and $b_{1} \ldots b_{r}=a_{1} \ldots a_{r}=a_{u n+1} \ldots a_{m}^{+}$, so

$$
a_{1} \ldots a_{m}=\left(b_{1} \ldots b_{n}\right)^{u} b_{1} \ldots b_{r}^{-} \text {. }
$$

By using that $s_{1} \ldots s_{m}=\mathbf{S}\left(a_{1} \ldots a_{m}\right)$ we obtain that

$$
\begin{aligned}
a_{1} & \ldots a_{m}^{+}\left(s_{1} \ldots s_{m}\right)^{\infty} \\
& =\left(b_{1} \ldots b_{n}\right)^{u} b_{1} \ldots b_{r}\left(s_{1} \ldots s_{m}\right)^{\infty} \\
& \preccurlyeq\left(b_{1} \ldots b_{n}\right)^{u} b_{1} \ldots b_{r}\left(b_{r+1} \ldots b_{n} b_{1} \ldots b_{r}^{-}\left(b_{1} \ldots b_{n}\right)^{u-1} b_{1} \ldots b_{r}\right)^{\infty} \\
& \prec\left(b_{1} \ldots b_{n}\right)^{\infty},
\end{aligned}
$$

leading to a contradiction with (4.2).

- If $n=m$, then by (4.2) we have $b_{1} \ldots b_{m}=a_{1} \ldots a_{m}$ or $b_{1} \ldots b_{m}=a_{1} \ldots a_{m}^{+}$. Both cases contradict (4.2). 
Therefore, we find that $n>m$. Write $n=k m+j$ with $k \geq 1$ and $1 \leq j \leq m$. By (4.2) we have

$$
b_{1} \ldots b_{n} \preccurlyeq a_{1} \ldots a_{m}^{+}\left(s_{1} \ldots s_{m}\right)^{k-1} s_{1} \ldots s_{j} .
$$

From

$$
s_{j+1} \ldots s_{m} s_{1} \ldots s_{j} \preccurlyeq a_{1} \ldots a_{m} \prec a_{1} \ldots a_{m}^{+}
$$

one can easily see that

$$
\left(a_{1} \ldots a_{m}^{+}\left(s_{1} \ldots s_{m}\right)^{k-1} s_{1} \ldots s_{j}\right)^{\infty} \succ a_{1} \ldots a_{m}^{+}\left(s_{1} \ldots s_{m}\right)^{\infty} \succcurlyeq\left(b_{1} \ldots b_{n}\right)^{\infty} .
$$

So, $b_{1} \ldots b_{n} \neq a_{1} \ldots a_{m}^{+}\left(s_{1} \ldots s_{m}\right)^{k-1} s_{1} \ldots s_{j}$ and hence

$$
b_{1} \ldots b_{n}^{+} \preccurlyeq a_{1} \ldots a_{m}^{+}\left(s_{1} \ldots s_{m}\right)^{k-1} s_{1} \ldots s_{j} .
$$

If strict inequality holds in (4.3), then (4.1) follows immediately and we are done. Suppose that the equality holds in (4.3). We split the proof of (4.1) into the following two cases. (I) $1 \leq j \leq(m / 2)$. Since $s_{1} \ldots s_{m}$ is a Lyndon word it follows that

$$
s_{1} \ldots s_{j}^{-} \prec s_{1} \ldots s_{j} \preccurlyeq s_{j+1} \ldots s_{2 j} .
$$

Furthermore, $t_{1} \ldots t_{n}$ is the Lyndon word for

$$
b_{1} \ldots b_{n}=a_{1} \ldots a_{m}^{+}\left(s_{1} \ldots s_{m}\right)^{k-1} s_{1} \ldots s_{j}^{-} .
$$

Then

$$
\begin{aligned}
\left(t_{1} \ldots t_{n}\right)^{\infty} & \preccurlyeq\left(s_{1} \ldots s_{j}^{-} a_{1} \ldots a_{m}^{+}\left(s_{1} \ldots s_{m}\right)^{k-1}\right)^{\infty} \\
& \prec\left(s_{j+1} \ldots s_{2 j} s_{2 j+1} \ldots s_{m} s_{1} \ldots s_{j}\right)^{\infty} .
\end{aligned}
$$

By (4.3) this proves (4.1), as required.

(II) $(m / 2)<j \leq m$. Since $s_{1} \ldots s_{m}$ and $t_{1} \ldots t_{n}$ are both Lyndon words, by Lemma 3.2(i) it follows that

$$
\begin{aligned}
\left(t_{1} \ldots t_{n}\right)^{\infty} & \preccurlyeq\left(s_{1} \ldots s_{m-j} s_{m-j+1} \ldots s_{j}^{-} a_{1} \ldots a_{m}^{+}\left(s_{1} \ldots s_{m}\right)^{k-1}\right)^{\infty} \\
& \prec\left(s_{j+1} \ldots s_{m} s_{1} \ldots s_{j}\right)^{\infty} .
\end{aligned}
$$

Again we have established (4.1).

4.1. Farey words. The set of Farey words is constructed recursively as follows. Let $F_{0}$ be the ordered set containing the two words 0 and 1 , i.e., $F_{0}:=(0,1)$. For each $n \geq 1$, $F_{n}=\left(v_{1}, \ldots, v_{2^{n}+1}\right)$ is the ordered set obtained from $F_{n-1}=\left(w_{1}, \ldots, w_{2^{n-1}+1}\right)$ by

$$
\begin{array}{ll}
v_{2 i-1}:=w_{i} & \text { for } 1 \leq i \leq 2^{n-1}+1, \\
v_{2 i} \quad:=w_{i} w_{i+1} & \text { for } 1 \leq i \leq 2^{n-1},
\end{array}
$$

where $w_{i} w_{i+1}$ denotes the concatenation of the words $w_{i}$ and $w_{i+1}$. For example,

$$
F_{0}=(0,1), \quad F_{1}=(0,01,1), \quad F_{2}=(0,001,01,011,1) .
$$

Then a word $w \in\{0,1\}^{*}$ is a Farey word if there is an $n \geq 0$ such that $\omega \in F_{n}$. For each $n \geq 0$ the words in $F_{n}$ are listed from left to right in a lexicographically increasing order (cf. [CIT18, Lemma 2.2]). In particular, no Farey word is periodic. Let

$$
\mathcal{F}:=\bigcup_{n \geq 0} F_{n} \backslash\{0,1\}
$$


be the set of non-degenerate Farey words. Clearly, any $w_{1} \ldots w_{m} \in \mathcal{F}$ has $w_{1}=0=$ $1-w_{m}$. It is well known that Farey words are balanced, i.e., if for $i=0,1$ we use $|u|_{i}$ to denote the number of occurrences of the symbol $i$ in the word $u$, then any $w \in \mathcal{F}$ has the property that for any two subwords $u$ and $v$ of $w$ of the same length and $i=0,1$, $\left.|| u\right|_{i}-|v|_{i} \mid \leq 1$. We recall from [CIT18, Proposition 2.3] the following definition.

Definition 4.2. Let $w=w_{1} \ldots w_{m} \in \mathcal{F}$. A decomposition $w=u v$ is called the standard factorization of $w$ if $u$ and $v$ are both Farey words.

By the construction of $F_{n}$ the standard factorization of a non-degenerate Farey word $w_{1} \ldots w_{m}$ is unique. We list some properties of Farey words. The proofs can be found in [CIT18, Propositions 2.8 and 2.9].

(f1) For $w_{1} \ldots w_{m} \in \mathcal{F}$ both $w_{1} \ldots w_{m-1} 0$ and $1 w_{2} \ldots w_{m}$ are palindromes, i.e.,

$$
w_{2} \ldots w_{m-1}=w_{m-1} \ldots w_{2} \text {. }
$$

(f2) Suppose that $w_{1} \ldots w_{m} \in \mathcal{F}$ has standard factorization $\left(w_{1} \ldots w_{m_{1}}\right)\left(w_{m_{1}+1} \ldots\right.$ $\left.w_{m}\right)$. The lexicographically largest cyclic permutation of $w_{1} \ldots w_{m}$ is given by

$$
w_{m-m_{1}+1} \ldots w_{m} w_{1} \ldots w_{m-m_{1}}=w_{m} w_{m-1} \ldots w_{2} w_{1} .
$$

(f3) Suppose that $w_{1} \ldots w_{m} \in \mathcal{F}$ has standard factorization $\left(w_{1} \ldots w_{m_{1}}\right)\left(w_{m_{1}+1} \ldots\right.$ $\left.w_{m}\right)$. Then $w_{1} \ldots w_{m}$ is a Lyndon word and its lexicographically second smallest cyclic permutation is $w_{m_{1}+1} \ldots w_{m} w_{1} \ldots w_{m_{1}}$.

Recall that for $w_{1} \ldots w_{m} \in\{0,1\}^{*}, \overline{w_{1} \ldots w_{m}}=\left(1-w_{1}\right)\left(1-w_{2}\right) \ldots\left(1-w_{m}\right)$ and note that by symmetry in the set $\mathcal{F}$,

$$
w_{1} \ldots w_{m} \in \mathcal{F} \Rightarrow \overline{w_{m} \ldots w_{1}} \in \mathcal{F} .
$$

By Lemma 3.2(i) it follows that if $w_{1} \ldots w_{m} \in \mathcal{F}$, then $\left(\overline{w_{1} \ldots w_{m}}\right)^{\infty} \in \mathcal{Q}$, i.e., $\sigma^{n}\left(\left(\overline{w_{1} \ldots w_{m}}\right)^{\infty}\right) \preccurlyeq\left(\overline{w_{1} \ldots w_{m}}\right)^{\infty}$ for all $n \geq 0$. Properties (f1), (f2) and (f3) imply the following.

LEMMA 4.3. Let $s_{1} \ldots s_{m} \in \mathcal{F}$ with $a_{1} \ldots a_{m}=\mathbf{L}\left(s_{1} \ldots s_{m}\right)$. Suppose that

$$
s_{1} \ldots s_{m}=\left(s_{1} \ldots s_{m_{1}}\right)\left(s_{m_{1}+1} \ldots s_{m}\right)
$$

is the standard factorization of $s_{1} \ldots s_{m}$.

(i) The words $a_{1} \ldots a_{m-1} 1$ and $0 a_{2} \ldots a_{m}$ are palindromes, i.e.,

$$
a_{2} \ldots a_{m-1}=a_{m-1} \ldots a_{2} .
$$

(ii) The Lyndon word associated to $a_{1} \ldots a_{m}$ is given by

$$
a_{m-m_{1}+1} \ldots a_{m} a_{1} \ldots a_{m-m_{1}}=a_{m} a_{m-1} \ldots a_{1} .
$$

(iii) $\left(a_{1} \ldots a_{m_{1}}\right)^{\infty} \in \mathcal{Q}$.

Proof. (i) and (ii) immediately follow from (f1) and (f2), respectively. For (iii) we know that $s_{1} \ldots s_{m_{1}}$ is a Lyndon word and therefore $\left(s_{1} \ldots s_{m_{1}}\right)^{\infty} \preccurlyeq \sigma^{n}\left(\left(s_{1} \ldots s_{m_{1}}\right)^{\infty}\right)$ for all $n \in \mathbb{N}$. This gives $\left(a_{1} \ldots a_{m_{1}}\right)^{\infty} \succcurlyeq \sigma^{n}\left(\left(a_{1} \ldots a_{m_{1}}\right)^{\infty}\right)$ for all $n \in \mathbb{N}$. 
For Farey words we obtain a strengthened version of Lemma 3.9, which will be useful in the proofs of Theorems $\mathrm{C}$ and D. We define a family $\left\{\Psi_{p}\right\}$ of substitutions first. For each $p \geq 1$ set

$$
\Psi_{p}(0)=0^{p+1} 1 \quad \text { and } \quad \Psi_{p}(1)=0^{p} 1 .
$$

We extend this definition to words $b_{1} \ldots b_{n} \in\{0,1\}^{*}$ by

$$
\Psi_{p}\left(b_{1} \ldots b_{n}\right)=\Psi_{p}\left(b_{1}\right) \ldots \Psi_{p}\left(b_{n}\right)
$$

and similarly for sequences in $\{0,1\}^{\mathbb{N}}$. One easily shows that $\tau_{k}$ preserves the lexicographical ordering $\{0,1\}^{\mathbb{N}}$ : for any two sequences $\left(b_{i}\right),\left(d_{i}\right) \in\{0,1\}^{\mathbb{N}}$ we have

$$
\left(b_{i}\right) \preccurlyeq\left(d_{i}\right) \Leftrightarrow \Psi_{p}\left(b_{i}\right) \preccurlyeq \Psi_{p}\left(d_{i}\right) .
$$

Proposition 4.4. Let $w=s_{1} \ldots s_{m} \in \mathcal{F}$. Then setting

$$
\mathcal{Z}_{w}:=\left\{\left(x_{i}\right) \in\{0,1\}^{\mathbb{N}}: w 0^{\infty} \preccurlyeq \sigma^{n}\left(\left(x_{i}\right)\right) \preccurlyeq\left(s_{m} \ldots s_{1}\right)^{\infty} \text { for all } n \geq 0\right\}
$$

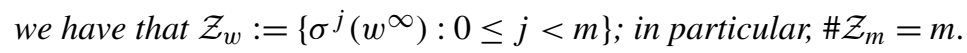

Proof. It is clear that $\left\{\sigma^{j}\left(w^{\infty}\right): 0 \leq j<m\right\} \subseteq \mathcal{Z}_{w}$. The other inclusion we prove by induction on the level of the Farey words. For $w=01$ the statement is trivial. Let $n \geq 2$ be given and assume that the statement is true for all non-degenerate Farey words of $F_{j}$, $j<n$. Let $w=s_{1} \ldots s_{m} \in F_{n}$. Note that if $w=0^{m-1} 1$ or $w=01^{m-1}$, then the statement is obviously true, so we exclude this case. Since all Farey words are balanced there is a $p$ such that $w$ is of the form

$$
w=0^{p+1} 10^{p_{1}} 1 \ldots 0^{p_{N}} 10^{p_{1}} 1 \text { or } \quad w=01^{p^{2}} 01^{p_{1}} \ldots 01^{p_{N}} 01^{p+1}
$$

for some $N \in \mathbb{N} \cup\{0\}$, where $p_{1} \ldots p_{N} \in\{p, p+1\}^{N}$ is a palindrome. Assume that $w=$ $0^{p+1} 10^{p_{1}} 1 \ldots 0^{p_{N}} 10^{p} 1$; the proof for the other case is similar. Recall the substitution $\Psi_{p}$ defined in (4.4). There is a word $v=0 t_{1} \ldots t_{N} 1 \in\{0,1\}^{*}$ with $\Psi_{p}(v)=w$. In [CIT18, Lemma 2.12] it is proven that $v$ is a Farey word, so $v \in F_{k}$ for some $k<n$. Moreover, since $w \neq 0^{p+1} 1$ we have $v \notin\{0,1\}$. Recall that

$$
s_{m} \ldots s_{1}=1 s_{2} \ldots s_{m-1} 0=10^{p} 10^{p_{1}} 1 \ldots 0^{p_{n}} 10^{p+1},
$$

so that

$$
\sigma\left(\left(s_{m} \ldots s_{1}\right)^{\infty}\right)=\Psi_{p}\left(\left(1 t_{1} \ldots t_{N} 0\right)^{\infty}\right)
$$

Let $x \in \mathcal{Z}_{w}$ be given. Then by the form of $w$ any two 1's in $x$ are separated by at least $p$ and at most $p+10$ 's. Assume first that $x_{1} \ldots x_{p+2}=0^{p+1} 1$, so that there is a $y \in\{0,1\}^{\mathbb{N}}$ such that $\Psi_{p}(y)=x$. Note that for any $r \geq 1$ there corresponds a $j \geq 1$ such that $\sigma^{j}(x)=$ $\Psi_{p}\left(\sigma^{r}(y)\right)$ since any digit in $y$ corresponds to a block $0^{p+1} 1$ or $0^{p} 1$ in $x$. From (3.4) we get that

$$
\Psi_{p}\left(\sigma^{r}(y)\right)=\sigma^{j}(x) \succcurlyeq w^{\infty}=\Psi_{p}\left(v^{\infty}\right),
$$

which by (4.5) above implies that $\sigma^{r}(y) \succcurlyeq v^{\infty}$ for all $r \geq 0$. On the other hand, from $\sigma^{j}(x) \preccurlyeq\left(s_{m} \ldots s_{1}\right)^{\infty}$ for all $j \geq 0$ it follows that $\sigma^{r}(y) \preccurlyeq\left(1 t_{2} \ldots t_{N} 0\right)^{\infty}$ for all $r \geq 0$. Hence, $y \in \mathcal{Z}_{v}$ and by the induction hypothesis there is an $\ell \in\{0,1, \ldots, N\}$ such that $y=\sigma^{\ell}\left(v^{\infty}\right)$. This implies that

$$
x=\Psi_{p}(y)=\sigma^{i}\left(w^{\infty}\right),
$$


where

$$
i= \begin{cases}0 & \text { if } \ell=0, \\ p+2 & \text { if } \ell=1, \\ (p+2)+\left(p_{1}+1\right)+\left(p_{2}+1\right)+\cdots+\left(p_{\ell-1}+1\right) & \text { if } 2 \leq \ell \leq N\end{cases}
$$

If $x$ is such that $x_{1} \ldots x_{j+1}=0^{j} 1$ for some $0 \leq j \leq p$, then there is a $y \in\{0,1\}^{\mathbb{N}}$ such that $\Psi_{p}(y)=\sigma^{j+1}(x)$ and by the same arguments as above we get that

$$
x=0^{j} 1 \sigma^{j+1}(x)=0^{j} 1 \Psi_{p}(y)=0^{j} 1 \sigma^{i}\left(w^{\infty}\right)=\sigma^{i^{\prime}}\left(w^{\infty}\right),
$$

where, in view of (4.6), $i^{\prime} \in\{0,1, \ldots, m-1\}$ is defined by

$$
i^{\prime}= \begin{cases}m-j-1 & \text { if } i=0 \\ i-j-1 & \text { otherwise. }\end{cases}
$$

This completes the proof.

4.2. Farey intervals. We now use the Farey words to identify the basic intervals $\left(\beta_{L}, \beta_{R}\right]$ that are not contained in any other basic interval.

Definition 4.5. Let $s_{1} \ldots s_{m} \in \mathcal{F}$ with $a_{1} \ldots a_{m}=\mathbf{L}\left(s_{1} \ldots s_{m}\right)$ and let $\gamma_{L}$ and $\gamma_{R}$ be given by the quasi-greedy expansions $\alpha\left(\gamma_{L}\right)=\left(a_{1} \ldots a_{m}\right)^{\infty}$ and $\alpha\left(\gamma_{R}\right)=$ $a_{1} \ldots a_{m}^{+}\left(a_{m} a_{m-1} \ldots a_{1}\right)^{\infty}$, respectively. Then the interval $J_{a_{1} \ldots a_{m}}=\left(\gamma_{L}, \gamma_{R}\right]$ is called the Farey interval generated by $a_{1} \ldots a_{m}$.

The following lemma is used to show that the Farey intervals are the maximal basic intervals.

LEMMA 4.6. Let $w=s_{1} \ldots s_{m} \in \mathcal{F}$ and let $a=a_{1} \ldots a_{m}=\mathbf{L}\left(s_{1} \ldots s_{m}\right)$. If $a_{n}=1$ for some $1 \leq n \leq m$, then

$$
\left(\mathbf{S}\left(a_{1} \ldots a_{n}^{-}\right)\right)^{\infty} \prec w^{\infty}
$$

Proof. We will prove this lemma by induction on the level of the Farey words. For the word 01 the statement is clear. Let $k \geq 2$ be given and assume that the statement holds for all non-degenerate Farey words in $F_{j}$ with $j<k$. Let $w=s_{1} \ldots s_{m} \in F_{k}$. If $w=0^{m-1} 1$ or $w=01^{m-1}$, then the statement obviously holds. Otherwise, in view of the fact that any Farey word is balanced, $w$ must have the form

$$
w=0^{p+1} 10^{p_{1}} 10^{p_{2}} \ldots 10^{p_{N}} 10^{p_{1}} 1 \text { or } \quad w=01^{p} 01^{p_{1}} 01^{p_{2}} \ldots 01^{p_{N}} 01^{p+1}
$$

for some $p \in \mathbb{N}$ and $N \in \mathbb{N} \cup\{0\}$, where $p_{1} \ldots p_{N} \in\{p, p+1\}^{N}$ is a palindrome. We split the proof into the following two cases.

(I) $w=0^{p+1} 10^{p_{1}} 10^{p_{2}} \ldots 10^{p_{N}} 10^{p} 1$. Then

$$
a=\mathbf{L}(w)=10^{p_{1}} 10^{p_{1}} 10^{p_{2}} \ldots 10^{p_{N}} 10^{p+1}=: 10^{p_{0}} 10^{p_{1}} 10^{p_{2}} \ldots 10^{p_{N}} 10^{p_{N+1}} .
$$

Let $\Psi_{p}$ be the substitution map from (4.4). Then by (4.7) there exists a word $v=$ $t_{0} t_{1} \ldots t_{N} t_{N+1}=1 t_{1} \ldots t_{N} 0$ such that

$$
\sigma\left(a^{\infty}\right)=\left(\Psi_{p}(v)\right)^{\infty} .
$$


By [CIT18, Lemma 2.12] it follows that $v=\mathbf{L}\left(0 t_{1} \ldots t_{N} 1\right)$ and $0 t_{1} \ldots t_{N} 1 \in F_{i}$ for some $i<k$. Let $1 \leq n \leq m$ be such that $a_{n}=1$. Then there is a $0 \leq j \leq N+1$ such that

$$
a_{1} \ldots a_{n}^{-}=10^{p_{0}} 10^{p_{1}} \ldots 10^{p_{j-1}} 10^{p_{j}+1} .
$$

Observe that $p_{j} \in\{p, p+1\}$. If $p_{j}=p+1$, then the Lyndon word $\mathbf{S}\left(a_{1} \ldots a_{n}^{-}\right)$begins with $0^{p+2} 1$ and $w$ begins with $0^{p+1} 1$. This implies that $\left(\mathbf{S}\left(a_{1} \ldots a_{n}^{-}\right)\right)^{\infty} \prec w^{\infty}$. If $p_{j}=$ $p$, then $t_{j}=1$ and

$$
\left(\mathbf{S}\left(a_{1} \ldots a_{n}^{-}\right)\right)^{\infty}=\left(\mathbf{S}\left(\Psi_{p}\left(1 t_{1} \ldots t_{j}^{-}\right)\right)\right)^{\infty} .
$$

By the induction hypothesis it follows that

$$
\left(\mathbf{S}\left(1 t_{1} \ldots t_{j}^{-}\right)\right)^{\infty} \prec\left(0 t_{1} \ldots t_{N} 1\right)^{\infty} .
$$

Since the map $\Psi_{p}$ preserves the lexicographical ordering (see (4.5)) this gives

$$
\begin{aligned}
\left(\mathbf{S}\left(a_{1} \ldots a_{n}^{-}\right)\right)^{\infty} & \left.=\left(\mathbf{S}\left(\Psi_{p}\left(1 t_{1} \ldots t_{j}^{-}\right)\right)\right)^{\infty}=\Psi_{p}\left(\mathbf{S}\left(1 t_{1} \ldots t_{j}^{-}\right)\right)^{\infty}\right) \\
& \prec \Psi_{p}\left(\left(0 t_{1} \ldots t_{N} 1\right)^{\infty}\right)=w^{\infty} .
\end{aligned}
$$

(II) $w=01^{p} 01^{p_{1}} 01^{p_{2}} \ldots 01^{p_{N}} 01^{p+1}$. Then the largest cyclic permutation of $w$ is

$$
a=\mathbf{L}(w)=1^{p+1} 01^{p_{1}} 01^{p_{2}} 0 \ldots 1^{p_{N}} 01^{p_{0}} 0=: 1^{p_{0}} 01^{p_{1}} 01^{p_{2}} 0 \ldots 1^{p_{N}} 01^{p_{N+1}} 0 .
$$

Define the substitution map $\hat{\Psi}_{p}$ by

$$
\hat{\Psi}_{p}(0)=01^{p} \quad \text { and } \quad \hat{\Psi}_{p}(1)=01^{p+1}
$$

and extend it to words and sequences in the usual way. One easily shows that $\hat{\Psi}_{p}$ preserves the lexicographical ordering. Then by (4.8) there exists a word $v=t_{0} t_{1} \ldots t_{N} t_{N+1}=$ $1 t_{1} \ldots t_{N} 0$ such that

$$
\sigma^{m-1}\left(a^{\infty}\right)=\hat{\Psi}_{p}\left(v^{\infty}\right) .
$$

Furthermore, by [CIT18, Lemma 2.12] it follows that $v=\mathbf{L}\left(0 t_{1} \ldots t_{N} 1\right)$ and $0 t_{1} \ldots t_{N}$ $1 \in F_{i}$ for some $i<k$. Let $1 \leq n \leq m$ be such that $a_{n}=1$. Then by (4.8) there exist $0 \leq j \leq N+1$ and $0<\ell \leq p_{j}$ such that

$$
a_{1} \ldots a_{n}^{-}=1^{p_{0}} 01^{p_{1}} 01^{p_{2}} 0 \ldots 1^{p_{j-1}} 01^{p_{j}-\ell} 0 .
$$

Observe that $p_{j} \in\{p, p+1\}$. Then $0 \leq p_{j}-\ell \leq p$. If $p_{j}-\ell<p$, then $\mathbf{S}\left(a_{1} \ldots a_{n}^{-}\right)$ begins with $01^{p_{j}-\ell} 0$ and $w$ begins with $01^{p}$. So, $\left(\mathbf{S}\left(a_{1} \ldots a_{n}^{-}\right)\right)^{\infty} \prec w^{\infty}$. If $p_{j}-\ell=p$, then $p_{j}=p+1$ and $t_{j}=1$. Since $0 t_{1} \ldots t_{N} 1$ is a non-degenerate Farey word in $F_{i}$ with $i<k$ by the induction hypothesis we have

$$
\left(\mathbf{S}\left(1 t_{1} \ldots t_{j}^{-}\right)\right)^{\infty} \prec\left(0 t_{1} \ldots t_{N} 1\right)^{\infty} .
$$

Since the map $\hat{\Psi}_{p}$ preserves the lexicographical ordering it follows that

$$
\begin{aligned}
\left(\mathbf{S}\left(a_{1} \ldots a_{n}^{-}\right)\right)^{\infty} & =\left(\mathbf{S}\left(\hat{\Psi}_{p}\left(1 t_{1} \ldots t_{j}^{-}\right)\right)\right)^{\infty}=\hat{\Psi}_{p}\left(\left(\mathbf{S}\left(1 t_{1} \ldots t_{j}^{-}\right)\right)^{\infty}\right) \\
& \prec \hat{\Psi}_{p}\left(\left(0 t_{1} \ldots t_{N} 1\right)^{\infty}\right)=w^{\infty} .
\end{aligned}
$$

This completes the lemma.

Proposition 4.7. Each Farey interval is a maximal basic interval. 
Proof. By Proposition 4.1 the basic intervals are nested, so it suffices to prove that a Farey interval cannot be contained in any other basic interval. Let $\left(\gamma_{L}, \gamma_{R}\right]$ be a Farey interval generated by a Farey word $s_{1} \ldots s_{m}$ and let $a_{1} \ldots a_{m}=\mathbf{L}\left(s_{1} \ldots s_{m}\right)$. Then

$$
\alpha\left(\gamma_{L}\right)=\left(a_{1} \ldots a_{m}\right)^{\infty} \text { and } \alpha\left(\gamma_{R}\right)=a_{1} \ldots a_{m}^{+}\left(s_{1} \ldots s_{m}\right)^{\infty} .
$$

Suppose on the contrary that there exists another basic interval $\left(\beta_{L}, \beta_{R}\right]$ such that $\left(\gamma_{L}, \gamma_{R}\right] \subsetneq\left(\beta_{L}, \beta_{R}\right]$. Assume that $\left(\beta_{L}, \beta_{R}\right]$ is generated by the Lyndon word $t_{1} \ldots t_{n}$ and let $b_{1} \ldots b_{n}=\mathbf{L}\left(t_{1} \ldots t_{n}\right)$. Then

$$
\alpha\left(\beta_{L}\right)=\left(b_{1} \ldots b_{n}\right)^{\infty} \text { and } \alpha\left(\beta_{R}\right)=b_{1} \ldots b_{n}^{+}\left(t_{1} \ldots t_{n}\right)^{\infty} .
$$

So, by using $\beta_{L}<\gamma_{L} \leq \beta_{R}$ it follows that

$$
\left(b_{1} \ldots b_{n}\right)^{\infty} \prec\left(a_{1} \ldots a_{m}\right)^{\infty} \preccurlyeq b_{1} \ldots b_{n}^{+}\left(t_{1} \ldots t_{n}\right)^{\infty} .
$$

By the same argument as in the proof of Proposition 4.1 we obtain $m>n$.

Now we claim that $a_{1} \ldots a_{n}=b_{1} \ldots b_{n}^{+}$. By (4.9) it follows that $b_{1} \ldots b_{n} \preccurlyeq$ $a_{1} \ldots a_{n} \preccurlyeq b_{1} \ldots b_{n}^{+}$. So, it suffices to prove that $a_{1} \ldots a_{n} \neq b_{1} \ldots b_{n}$. Suppose that $a_{1} \ldots a_{n}=b_{1} \ldots b_{n}$. Write $m=k n+j$ with $k \geq 1$ and $1 \leq j \leq n$. Note that $a_{1} \ldots a_{m}$ is the largest cyclic permutation of a Farey word. Then $a_{i+1} \ldots a_{m} \prec a_{1} \ldots a_{m-i}$ for all $i<m$. So,

$$
a_{1} \ldots a_{m} \preccurlyeq\left(a_{1} \ldots a_{n}\right)^{k} a_{m-j+1} \ldots a_{m} \prec\left(a_{1} \ldots a_{n}\right)^{k} a_{1} \ldots a_{j}=\left(b_{1} \ldots b_{n}\right)^{k} b_{1} \ldots b_{j},
$$

leading to a contradiction with (4.9). This establishes the claim.

By the claim it follows that $a_{n}=1$ and $t_{1} \ldots t_{n}=\mathbf{S}\left(b_{1} \ldots b_{n}\right)=\mathbf{S}\left(a_{1} \ldots a_{n}^{-}\right)$. Since $s_{1} \ldots s_{m}$ is a non-degenerate Farey word and $a_{1} \ldots a_{m}=\mathbf{L}\left(s_{1} \ldots s_{m}\right)$, by Lemma 4.6 it follows that

$$
\left(t_{1} \ldots t_{n}\right)^{\infty} \prec\left(s_{1} \ldots s_{m}\right)^{\infty} \preccurlyeq\left(a_{n+1} \ldots a_{m} a_{1} \ldots a_{n}\right)^{\infty} .
$$

Again by the claim we conclude that

$$
b_{1} \ldots b_{n}^{+}\left(t_{1} \ldots t_{n}\right)^{\infty} \prec\left(a_{1} \ldots a_{m}\right)^{\infty} .
$$

This leads to a contradiction with (4.9).

Proposition 3.10 states that for any $\beta \in J_{a_{1} \ldots a_{m}}$ the set $E_{\beta}^{+}$contains an isolated point. So, the set of $\beta \in(1,2)$ for which $E_{\beta}^{+}$has no isolated points is a subset of $(1,2) \backslash \bigcup_{s_{1} \ldots s_{m} \in \mathcal{F}} J_{s_{m} \ldots s_{1}}$. Suppose on the other hand that $\beta \in(1,2) \backslash \bigcup_{s_{1} \ldots s_{m} \in \mathcal{F}} J_{s_{m} \ldots s_{1}}$. From Proposition 3.3 we know that any isolated point $t$ of $E_{\beta}^{+}$must have a periodic $\beta$ expansion $b(t, \beta)$. To such a $\beta$-expansion we can relate a basic interval $\left(\beta_{L}, \beta_{R}\right]$ as in Proposition 3.10. From the maximality of the Farey intervals and Proposition 3.10 we can then deduce that $t$ is not isolated for $E_{\beta}^{+}$. Thus, the set of $\beta \in(1,2)$ for which $E_{\beta}^{+}$has no isolated points is in fact equal to the set

$$
(1,2) \backslash \bigcup_{s_{1} \ldots s_{m} \in \mathcal{F}} J_{s_{m} \ldots s_{1}} \text {. }
$$


To prove Theorem $\mathrm{C}$ it is therefore enough to prove that this set has Hausdorff dimension zero. We do so by relating each Farey interval $J_{a_{1} \ldots a_{m}}$ to another interval $I_{\overline{a_{1} \ldots a_{m}}}$ associated to the doubling map and using known results for the union $\bigcup I \overline{a_{1} \ldots a_{m}}$.

Recall that the doubling map is given by $T_{2}(x)=2 x(\bmod 1)$ and that $\pi_{2}:\{0,1\}^{\mathbb{N}} \rightarrow$ $[0,1]$ is the projection map defined in (2.1). Set

$$
E_{D}:=\left\{x \in\left[0, \frac{1}{2}\right): T_{2}^{n}(x) \in\left[x, x+\frac{1}{2}\right] \text { for all } n \geq 0\right\} .
$$

For each Farey word $w=w_{1} \ldots w_{m} \in \mathcal{F}$ we denote by $I_{w}:=\left(q_{L}, q_{R}\right)$ the open interval associated to $w$, where

$$
q_{L}=\pi_{2}\left(\left(w_{m} w_{m-1} \ldots w_{1}\right)^{\infty}\right)-\frac{1}{2} \quad \text { and } \quad q_{R}=\pi_{2}\left(\left(w_{1} \ldots w_{m}\right)^{\infty}\right) .
$$

The interval $I_{w}=\left(q_{L}, q_{R}\right)$ is well defined since by (f1) it follows that

$$
\begin{aligned}
q_{L} & =\pi_{2}\left(0 w_{m-1} w_{m-2} \ldots w_{1}\left(w_{m} w_{m-1} \ldots w_{1}\right)^{\infty}\right) \\
& =\pi_{2}\left(w_{1} w_{2} \ldots w_{m-1} 0\left(w_{m} w_{m-1} \ldots w_{1}\right)^{\infty}\right)<\pi_{2}\left(\left(w_{1} \ldots w_{m}\right)^{\infty}\right)=q_{R} .
\end{aligned}
$$

In [CIT18] we find the following result.

Proposition 4.8. [CIT18, Proposition 2.14]

(i) Each $I_{w}$ is a connected component of $\left(0, \frac{1}{2}\right) \backslash E_{D}$. Moreover,

$$
\left(0, \frac{1}{2}\right) \backslash E_{D}=\bigcup_{w \in \mathcal{F}} I_{w}
$$

(ii) $\operatorname{dim}_{H} E_{D}=0$.

Recall that by Lemma 2.1 the function $\alpha: \beta \mapsto \alpha(\beta)$ is a strictly increasing bijection from $(1,2]$ to $\mathcal{Q}$. Moreover, $\pi_{2}:\{0,1\}^{\mathbb{N}} \rightarrow(0,1]$ is a strictly increasing bijection if we remove from $\{0,1\}^{\mathbb{N}}$ all sequences ending with $0^{\infty}$. Since such sequences do not occur as quasi-greedy expansions of 1 and since the first digit $\alpha_{1}(\beta)$ equals 1 for any $\beta \in(1,2)$, the map

$$
\phi:(1,2) \rightarrow\left(\frac{1}{2}, 1\right), \beta \mapsto \pi_{2}(\alpha(\beta))=\sum_{i=1}^{\infty} \frac{\alpha_{i}(\beta)}{2^{i}}
$$

is strictly increasing as well. The image $\phi((1,2))$ is a proper subset of $\left(\frac{1}{2}, 1\right)$.

LEMMA 4.9.

$$
\phi\left((1,2) \backslash \bigcup_{s_{1} \ldots s_{m} \in \mathcal{F}} J_{s_{m} \ldots s_{1}}\right) \subseteq\left(\frac{1}{2}, 1\right) \backslash \bigcup_{s_{1} \ldots s_{m} \in \mathcal{F}}\left(1-I_{s_{1} \ldots s_{m}}\right)=1-E_{D} .
$$

Proof. Let $s_{1} \ldots s_{m} \in \mathcal{F}$ with $a_{1} \ldots a_{m}=\mathbf{L}\left(s_{1} \ldots s_{m}\right)$. Note that

$$
q_{R}=\pi_{2}\left(\left(\overline{a_{1} \ldots a_{m}}\right)^{\infty}\right)=\sum_{n \geq 1} \frac{1}{2^{n}}-\pi_{2}\left(\left(a_{1} \ldots a_{m}\right)^{\infty}\right)=1-\phi\left(\gamma_{L}\right) .
$$

Moreover, by Lemma 4.3(i) and (ii) it follows that

$$
\alpha\left(\gamma_{R}\right)=a_{1} \ldots a_{m}^{+}\left(a_{m} a_{m-1} \ldots a_{1}\right)^{\infty}=1 a_{m-1} a_{m-2} \ldots a_{1}\left(a_{m} a_{m-1} \ldots a_{1}\right)^{\infty} .
$$


Then

$$
\begin{aligned}
\phi\left(\gamma_{R}\right) & =\pi_{2}\left(1 a_{m-1} a_{m-2} \ldots a_{1}\left(a_{m} a_{m-1} \ldots a_{1}\right)^{\infty}\right) \\
& =\frac{1}{2}+\pi_{2}\left(\left(a_{m} a_{m-1} \ldots a_{1}\right)^{\infty}\right)=\frac{1}{2}+\left(1-\pi_{2}\left(\left(\overline{a_{m} a_{m-1} \ldots a_{1}}\right)^{\infty}\right)\right) \\
& =1-\left(\pi_{2}\left(\left(\overline{a_{m} a_{m-1} \ldots a_{1}}\right)^{\infty}\right)-\frac{1}{2}\right)=1-q_{L} .
\end{aligned}
$$

Since $\phi$ is strictly increasing and bijective from $(1,2)$ to $\phi((1,2))$ this implies that

$$
\phi^{-1}\left(\left(1-q_{R}, 1-q_{L}\right)\right)=\left(\gamma_{L}, \gamma_{R}\right) \text {. }
$$

By Proposition 4.8(i) this gives the result.

Finally, to determine the Hausdorff dimension of $(1,2) \backslash \bigcup_{s_{1} \ldots s_{m} \in \mathcal{F}} J_{s_{m} \ldots s_{1}}$, we prove that the inverse $\phi^{-1}: \pi_{2} \circ \alpha((1,2)) \rightarrow(1,2)$ is Hölder continuous and combine this with the following well-known result: if $f:\left(X, \rho_{1}\right) \rightarrow\left(Y, \rho_{2}\right)$ is a $c$-Hölder continuous map between two metric spaces $\left(X, \rho_{1}\right)$ and $\left(Y, \rho_{2}\right)$, then $\operatorname{dim}_{H} f(X) \leq(1 / c) \operatorname{dim}_{H} X$.

LEMMA 4.10. For any integer $N \geq 2$ the function $\phi^{-1}$ is $c$-Hölder continuous with $c=$ $(\log (1+1 / N) / \log 4)$ on the set $\phi([1+(1 / N), 2))$.

Proof. Fix $N \geq 2$ and let $\beta_{1}, \beta_{2} \in[1+(1 / N), 2)$ with $\beta_{1}<\beta_{2}$. Then $\alpha\left(\beta_{1}\right) \prec \alpha\left(\beta_{2}\right)$. Let $n$ be the positive integer such that

$$
\alpha_{1}\left(\beta_{1}\right) \ldots \alpha_{n-1}\left(\beta_{1}\right)=\alpha_{1}\left(\beta_{2}\right) \ldots \alpha_{n-1}\left(\beta_{2}\right) \text { and } \alpha_{n}\left(\beta_{1}\right)<\alpha_{n}\left(\beta_{2}\right) .
$$

By using $1=\pi_{\beta_{1}}\left(\alpha\left(\beta_{1}\right)\right)=\pi_{\beta_{2}}\left(\alpha\left(\beta_{2}\right)\right)$ and (4.10) it follows that

$$
\begin{aligned}
0<\beta_{2}-\beta_{1} & =\beta_{2} \sum_{j=1}^{\infty} \frac{\alpha_{j}\left(\beta_{2}\right)}{\beta_{2}^{j}}-\beta_{1} \sum_{j=1}^{\infty} \frac{\alpha_{j}\left(\beta_{1}\right)}{\beta_{1}^{j}} \\
& \leq \sum_{j=1}^{\infty} \frac{\alpha_{j}\left(\beta_{2}\right)}{\beta_{2}^{j-1}}-\sum_{j=1}^{\infty} \frac{\alpha_{j}\left(\beta_{1}\right)}{\beta_{2}^{j-1}} \\
& =\sum_{j=n}^{\infty} \frac{\alpha_{j}\left(\beta_{2}\right)-\alpha_{j}\left(\beta_{1}\right)}{\beta_{2}^{j-1}} \leq \sum_{j=n}^{\infty} \frac{1}{\left(1+\frac{1}{N}\right)^{j-1}}=N\left(1+\frac{1}{N}\right)^{2-n} .
\end{aligned}
$$

On the other hand, by (4.10) we also have

$$
\begin{aligned}
\pi_{2}\left(\alpha\left(\beta_{2}\right)\right)-\pi_{2}\left(\alpha\left(\beta_{1}\right)\right) & =\sum_{j=1}^{\infty} \frac{\alpha_{j}\left(\beta_{2}\right)-\alpha_{j}\left(\beta_{1}\right)}{2^{j}}=\sum_{j=n}^{\infty} \frac{\alpha_{j}\left(\beta_{2}\right)-\alpha_{j}\left(\beta_{1}\right)}{2^{j}} \\
& \geq \frac{1}{2^{n}}-\sum_{j=n+1}^{\infty} \frac{\alpha_{j}\left(\beta_{1}\right)}{2^{j}} \geq \frac{1}{2^{n}\left(2^{n}-1\right)}>\frac{1}{4^{n}},
\end{aligned}
$$

where the second inequality follows by Lemma 2.1 and the fact that

$$
\alpha_{n+1}\left(\beta_{1}\right) \alpha_{n+2}\left(\beta_{1}\right) \cdots \preccurlyeq \alpha_{1}\left(\beta_{1}\right) \alpha_{2}\left(\beta_{1}\right) \cdots \preccurlyeq\left(1^{n-1} 0\right)^{\infty} .
$$

Combining (4.11) and (4.12) we conclude that

$$
\begin{aligned}
\left|\pi_{2}\left(\alpha\left(\beta_{2}\right)\right)-\pi_{2}\left(\alpha\left(\beta_{1}\right)\right)\right| \geq & \frac{1}{4^{n}}=\left(1+\frac{1}{N}\right)^{-(\log 4) /(\log (1+1 / N)) n} \\
\geq & \left(N(1+1 / N)^{2}\right)^{-(\log 4) /(\log (1+1 / N))} \\
& \times\left|\beta_{2}-\beta_{1}\right|^{(\log 4) /(\log (1+1 / N))} .
\end{aligned}
$$


Proof of Theorem C. By Lemma 4.9 the only thing left to show is that $\operatorname{dim}_{H} \phi^{-1}(1-$ $\left.E_{D}\right)=0$. This follows from Lemma 4.10 and Proposition 4.8(ii) in the following way:

$$
\begin{aligned}
0 & \leq \operatorname{dim}_{H} \phi^{-1}\left(1-E_{D}\right)=\operatorname{dim}_{H}\left(\bigcup_{N \geq 2}\left(\phi^{-1}\left(1-E_{D}\right) \cap\left[1+\frac{1}{N}, 2\right)\right)\right) \\
& =\sup _{N \geq 2} \operatorname{dim}_{H} \phi^{-1}\left(\left(1-E_{D}\right) \cap \phi\left(\left[1+\frac{1}{N}, 2\right)\right)\right) \\
& \leq \sup _{N \geq 2} \frac{\log 4}{\log (1+1 / N)} \operatorname{dim}_{H}\left(\left(1-E_{D}\right) \cap \phi\left(\left[1+\frac{1}{N}, 2\right)\right)\right) \\
& \leq \sup _{N \geq 2} \frac{\log 4}{\log (1+1 / N)} \operatorname{dim}_{H}\left(1-E_{D}\right)=\sup _{N \geq 2} \frac{\log 4}{\log (1+1 / N)} \operatorname{dim}_{H} E_{D}=0 .
\end{aligned}
$$

\section{The critical points of the dimension function}

Since the map $\eta_{\beta}: t \mapsto \operatorname{dim}_{H} K_{\beta}(t)$ is a decreasing, continuous function with $\eta_{\beta}(0)=1$ and $\eta_{\beta}(1 / \beta)=0$, there is a unique value $\tau_{\beta}$ such that $\operatorname{dim}_{H} K_{\beta}(t)>0$ if and only if $t<\tau_{\beta}$. Determining the value of $\tau_{\beta}$ would extend the results from [Cla16] for holes of the form $(0, t)$. For $\beta=\gamma_{L}$ equal to the left end point of one of the Farey intervals, we show below that $\tau_{\beta}=1-(1 / \beta)$. This result is based on the following lemma.

LEMMA 5.1. Let $s_{1} \ldots s_{m} \in \mathcal{F}$ with $a_{1} \ldots a_{m}=\mathbf{L}\left(s_{1} \ldots s_{m}\right)$. Let $1 \leq j \leq m$ be such that $s_{1} \ldots s_{m}=a_{j+1} \ldots a_{m} a_{1} \ldots a_{j}$. For each $N \geq 1$ define the sequence $\mathbf{t}_{N} \in\{0,1\}^{\mathbb{N}}$ by

$$
\mathbf{t}_{N}:=\left(0 a_{2} \ldots a_{m}\left(a_{1} \ldots a_{m}\right)^{N} a_{1} \ldots a_{j}\right)^{\infty} .
$$

Then for each $N \geq 1, \mathbf{t}_{N} \prec \mathbf{t}_{N+1}$. Furthermore, any sequence $\mathbf{t}$ that is a concatenation of blocks of the form

$$
0 a_{2} \ldots a_{m}\left(a_{1} \ldots a_{m}\right)^{k} a_{1} \ldots a_{j}, \quad k \geq N,
$$

satisfies $\mathbf{t}_{N} \preccurlyeq \sigma^{n}(\mathbf{t}) \prec\left(a_{1} \ldots a_{m}\right)^{\infty}$ for all $n \geq 0$. In particular, we have for each $n \geq 0$ that

$$
\mathbf{t}_{N} \preccurlyeq \sigma^{n}\left(\mathbf{t}_{N}\right) \prec\left(a_{1} \ldots a_{m}\right)^{\infty}
$$

Proof. By Lemma 4.3 it follows that

$$
s_{1} \ldots s_{m}=a_{m} a_{m-1} \ldots a_{1}=0 a_{2} \ldots a_{m}^{+}=a_{j+1} \ldots a_{m} a_{1} \ldots a_{j} .
$$

This implies that for all $N \geq 1$

$$
\begin{aligned}
\mathbf{t}_{N} & =\left(0 a_{2} \ldots a_{m}\left(a_{1} \ldots a_{m}\right)^{N} a_{1} \ldots a_{j}\right)\left(a_{j+1} \ldots a_{m} a_{1} \ldots a_{j}^{-}\left(a_{1} \ldots a_{m}\right)^{N} a_{1} \ldots a_{j}\right)^{\infty} \\
& \prec\left(0 a_{2} \ldots a_{m}\left(a_{1} \ldots a_{m}\right)^{N+1} a_{1} \ldots a_{j}\right)^{\infty}=\mathbf{t}_{N+1}
\end{aligned}
$$

giving the first part of the statement. For the second part, let $\mathbf{t}$ be a sequence consisting of a concatenation of blocks of the form $0 a_{2} \ldots a_{m}\left(a_{1} \ldots a_{m}\right)^{k} a_{1} \ldots a_{j}$ with prefix $0 a_{2} \ldots a_{m}\left(a_{1} \ldots a_{m}\right)^{K} a_{1} \ldots a_{j}$ for some $K \geq N$. We first show that $\sigma^{n}(\mathbf{t}) \prec$ $\left(a_{1} \ldots a_{m}\right)^{\infty}$ for all $n \geq 0$. For $n=0$ the statement is clear. By Lemma 3.8 it follows that $a_{i+1} \ldots a_{m} \prec a_{1} \ldots a_{m-i}$ for each $0<i<m$. This implies that $\sigma^{n}(\mathbf{t}) \prec\left(a_{1} \ldots a_{m}\right)^{\infty}$ for 
each $\ell m<n<(\ell+1) m, 0 \leq \ell \leq K$. For all other values of $n<(K+1) m+j$ we obtain the result from (5.2), which implies that

$$
a_{1} \ldots a_{j} 0 a_{2} \ldots a_{m}=a_{1} \ldots a_{m} a_{1} \ldots a_{j}^{-} \prec a_{1} \ldots a_{m} a_{1} \ldots a_{j} .
$$

The same arguments then give the result for any $n \geq 0$. Hence, $\sigma^{n}(\mathbf{t}) \prec\left(a_{1} \ldots a_{m}\right)^{\infty}$ for all $n \geq 0$. We now show that $\sigma^{n}(\mathbf{t}) \succcurlyeq \mathbf{t}_{N}$ for each $n \geq 0$. Note that $\mathbf{t}$ has prefix

$$
s_{1} \ldots s_{m}^{-}\left(a_{1} \ldots a_{m}\right)^{K} a_{1} \ldots a_{j} .
$$

For $n=0$ the statement follows from (5.2). By (5.2) and Lemmas 3.2 and 3.8 it follows that

$$
s_{i+1} \ldots s_{m}^{-} \succcurlyeq s_{1} \ldots s_{m-i} \quad \text { and } \quad a_{1} \ldots a_{i} \succ a_{m-i+1} \ldots a_{m}=s_{m-i+1} \ldots s_{m}^{-}
$$

for all $0<i<m$, giving the statement for all $0<n<m$. Since $s_{1} \ldots s_{m}$ is the Lyndon word associated to $a_{1} \ldots a_{m}$ we obtain

$$
a_{i+1} \ldots a_{m} a_{1} \ldots a_{i} \succcurlyeq s_{1} \ldots s_{m} \succ s_{1} \ldots s_{m}^{-} \text {for any } 0 \leq i<m .
$$

Since $a_{1} \ldots a_{j} s_{1} \ldots s_{m-j}=a_{1} \ldots a_{m}$ the conclusion that $\sigma^{n}(\mathbf{t}) \succcurlyeq \mathbf{t}_{N}$ for all $n \geq 0$ follows.

Proposition 5.2. Let $s_{1} \ldots s_{m} \in \mathcal{F}$ with $a_{1} \ldots a_{m}=\mathbf{L}\left(s_{1} \ldots s_{m}\right)$ and let $\beta \in(1,2)$ be such that $\alpha(\beta)=\left(a_{1} \ldots a_{m}\right)^{\infty}$. Then $1-(1 / \beta) \in E_{\beta}^{0}$ and

$$
\tau_{\beta}=1-\frac{1}{\beta}=\max \overline{E_{\beta}^{+}} .
$$

Proof. Since $m$ is the minimal period of $\alpha(\beta)$ the greedy $\beta$-expansion of 1 is equal to $b(1, \beta)=a_{1} \ldots a_{m}^{+} 0^{\infty}$. Lemma 4.3 tells us that $a_{1} \ldots a_{m}^{+}=1 a_{m-1} \ldots a_{1}$, so

$$
\pi_{\beta}\left(a_{m} a_{m-1} \ldots a_{1} 0^{\infty}\right)=\pi_{\beta}\left(1 a_{m-1} \ldots a_{1} 0^{\infty}\right)-\frac{1}{\beta}=\pi_{\beta}\left(a_{1} \ldots a_{m}^{+} 0^{\infty}\right)-\frac{1}{\beta}=1-\frac{1}{\beta} .
$$

Recall that $a_{m} a_{m-1} \ldots a_{1}=0 a_{2} \ldots a_{m}^{+}$. Then by Lemma 3.8 it follows that for each $n \geq 0, \quad \sigma^{n}\left(a_{m} a_{m-1} \ldots a_{1} 0^{\infty}\right) \prec\left(a_{1} \ldots a_{m}\right)^{\infty}=\alpha(\beta)$ and hence $a_{m} a_{m-1} \ldots a_{1} 0^{\infty}$ is the greedy $\beta$-expansion of $1-(1 / \beta)$, i.e., $b(1-(1 / \beta), \beta)=a_{m} a_{m-1} \ldots a_{1} 0^{\infty}$. By Lemma $3.2, b(1-(1 / \beta), \beta) \in \mathcal{E}_{\beta}^{0}$, so $1-(1 / \beta) \in E_{\beta}^{0}$.

The quasi-greedy $\beta$-expansion of $1-(1 / \beta)$ is given by

$$
\tilde{b}\left(1-\frac{1}{\beta}, \beta\right)=0 a_{2} \ldots a_{m}\left(a_{1} \ldots a_{m}\right)^{\infty} .
$$

Now consider the sequences $\mathbf{t}_{N}$ from Lemma 5.1. Since $\mathbf{t}_{N} \preccurlyeq \sigma^{n}\left(\mathbf{t}_{N}\right) \prec\left(a_{1} \ldots a_{m}\right)^{\infty}=$ $\alpha(\beta)$ for all $n \geq 0$ we have $\mathbf{t}_{N} \in \mathcal{E}_{\beta}^{+}$for each $N \geq 1$. Moreover, if we set $t_{N}:=$ $\pi_{\beta}\left(\mathbf{t}_{N}\right)$, then Lemma 2.2 gives that $t_{N} \nearrow 1-(1 / \beta)$ as $N \rightarrow \infty$. So, $\max \overline{E_{\beta}^{+}} \geq 1-$ $(1 / \beta)$. Furthermore, the fact that any sequence of concatenations of blocks of the form $0 a_{2} \ldots a_{m}\left(a_{1} \ldots a_{m}\right)^{k} a_{1} \ldots a_{j}, k \geq N$, belongs to $\mathcal{K}_{\beta}^{+}\left(t_{N}\right)$ implies that $h_{\text {top }}\left(\mathcal{K}_{\beta}^{+}\left(t_{N}\right)\right)$ $>0$ for all $N \geq 1$ and hence also $h_{\text {top }}\left(\mathcal{K}_{\beta}\left(t_{N}\right)\right)>0$ for all $N \geq 1$. By the dimension formula (2.6) we then get that $\tau_{\beta} \geq 1-(1 / \beta)$. 
On the other hand, by Lemma 4.3(ii) and Proposition 4.4 we have

$$
\mathcal{K}_{\beta}^{+}\left(1-\frac{1}{\beta}\right)=\left\{\left(x_{i}\right): a_{m} a_{m-1} \ldots a_{1} 0^{\infty} \preccurlyeq \sigma^{n}\left(\left(x_{i}\right)\right) \prec\left(a_{1} \ldots a_{m}\right)^{\infty} \forall n \geq 0\right\}=\emptyset .
$$

Since $E_{\beta}^{+} \cap[1-(1 / \beta), 1] \subseteq K_{\beta}^{+}(1-(1 / \beta))$ this implies that $\max \overline{E_{\beta}^{+}} \leq 1-(1 / \beta)$. It also implies that $\operatorname{dim}_{H} K_{\beta}(1-(1 / \beta))=0$, which gives that $\tau_{\beta} \leq 1-(1 / \beta)$ and proves the result.

Remark 5.3. Note that the previous lemma also implies that for any $t<1-\left(1 / \gamma_{L}\right)$ we have $h_{\text {top }}\left(\mathcal{K}_{\gamma_{L}}^{+}(t)\right)>0$. We will use this later on.

Next we will give a lower and an upper bound for $\tau_{\beta}$ on each Farey interval $\left(\gamma_{L}, \gamma_{R}\right]$.

LEMMA 5.4. Let $s_{1} \ldots s_{m} \in \mathcal{F}$ with $a_{1} \ldots a_{m}=\mathbf{L}\left(s_{1} \ldots s_{m}\right)$. For each $\beta \in\left(\gamma_{L}, \gamma_{R}\right]$ set $t^{*}=\pi_{\beta}\left(0 a_{2} \ldots a_{m}\left(a_{1} \ldots a_{m}\right)^{\infty}\right)$ and $t^{\diamond}=\pi_{\beta}\left(0 a_{2} \ldots a_{m}^{+} 0^{\infty}\right)$. Then $t^{*} \in E_{\beta}^{+}, t^{\diamond} \in E_{\beta}^{0}$ and

$$
1-\frac{1}{\beta}-\frac{1}{\beta^{m}}+\frac{1}{\beta\left(\beta^{m}-1\right)} \leq t^{*} \leq \tau_{\beta} \leq t^{\diamond}<1-\frac{1}{\beta}
$$

Proof. Take $\beta \in\left(\gamma_{L}, \gamma_{R}\right]$. Then

$$
\left(a_{1} \ldots a_{m}\right)^{\infty} \prec \alpha(\beta) \preccurlyeq a_{1} \ldots a_{m}^{+}\left(a_{m} a_{m-1} \ldots a_{1}\right)^{\infty} .
$$

We first show that $\tau_{\beta} \geq t^{*}$. By Lemmas 4.3 and 3.8 we have

$$
\sigma^{n}\left(0 a_{2} \ldots a_{m}\left(a_{1} \ldots a_{m}\right)^{\infty}\right) \preccurlyeq\left(a_{1} \ldots a_{m}\right)^{\infty} \prec \alpha(\beta) \text { for all } n \geq 0 .
$$

Hence, $b\left(t^{*}, \beta\right)=0 a_{2} \ldots a_{m}\left(a_{1} \ldots a_{m}\right)^{\infty}$ and as in the proof of Lemma 5.1 we have that $\sigma^{n}\left(b\left(t^{*}, \beta\right)\right) \succcurlyeq b\left(t^{*}, \beta\right)$ for each $n \geq 0$. So, $t^{*} \in E_{\beta}^{+}$.

For each $t<t^{*}$ we have by Lemma 2.2 that $b(t, \beta) \prec 0 a_{2} \ldots a_{m}\left(a_{1} \ldots a_{m}\right)^{\infty}$. This implies that for $N$ large enough $b(t, \beta) \prec \mathbf{t}_{N} \prec\left(a_{1} \ldots a_{m}\right)^{\infty} \prec \alpha(\beta)$. By Lemma 5.1 it follows that $t_{N} \in \mathcal{K}_{\beta}^{+}(t)$ and $h_{\text {top }}\left(\mathcal{K}_{\beta}(t)\right) \geq h_{\text {top }}\left(\mathcal{K}_{\beta}^{+}(t)\right)>0$. Thus, $\operatorname{dim}_{H} \mathcal{K}_{\beta}(t)>0$ and $\tau_{\beta} \geq t^{*}$.

On the other hand, for $t^{\diamond}$ we have that $0 a_{2} \ldots a_{m}^{+} 0^{\infty}$ is admissible for any $\beta \in$ $\left(\gamma_{L}, \gamma_{R}\right]$ and that $\sigma^{n}\left(0 a_{2} \ldots a_{m}^{+} 0^{\infty}\right) \succ 0 a_{2} \ldots a_{m}^{+} 0^{\infty}$ for all $0<n<m$, so $t^{\diamond} \in E_{\beta}^{0}$. By Lemmas 4.3 and 3.7 we get

$$
\begin{aligned}
\mathcal{K}_{\beta}^{+} & \left(t^{\diamond}\right) \\
& \subseteq\left\{\left(x_{i}\right): a_{m} a_{m-1} \ldots a_{1} 0^{\infty} \preccurlyeq \sigma^{n}\left(\left(x_{i}\right)\right) \prec a_{1} \ldots a_{m}^{+}\left(a_{m} a_{m-1} \ldots a_{1}\right)^{\infty} \forall n \geq 0\right\} \\
& =\left\{\left(x_{i}\right):\left(a_{m} a_{m-1} \ldots a_{1}\right)^{\infty} \preccurlyeq \sigma^{n}\left(\left(x_{i}\right)\right) \prec a_{1} \ldots a_{m}^{+}\left(a_{m} a_{m-1} \ldots a_{1}\right)^{\infty} \forall n \geq 0\right\} \\
& =\left\{\left(x_{i}\right):\left(a_{m} a_{m-1} \ldots a_{1}\right)^{\infty} \preccurlyeq \sigma^{n}\left(\left(x_{i}\right)\right) \preccurlyeq\left(a_{1} \ldots a_{m}\right)^{\infty} \forall n \geq 0\right\} \\
& =\left\{\left(x_{i}\right): a_{m} a_{m-1} \ldots a_{1} 0^{\infty} \preccurlyeq \sigma^{n}\left(\left(x_{i}\right)\right) \preccurlyeq\left(a_{1} \ldots a_{m}\right)^{\infty} \forall n \geq 0\right\} .
\end{aligned}
$$

By Proposition 4.4 it follows that $\# \mathcal{K}_{\beta}^{+}\left(t^{\diamond}\right)<\infty$, so that $\operatorname{dim}_{H} K_{\beta}\left(t^{\diamond}\right)=0$. This gives that $\tau_{\beta} \leq t^{\diamond}$. Note that

$$
\pi_{\gamma_{R}}\left(a_{1} a_{2} \ldots a_{m}^{+}\left(0 a_{2} \ldots a_{m}\right)^{\infty}\right)=1
$$




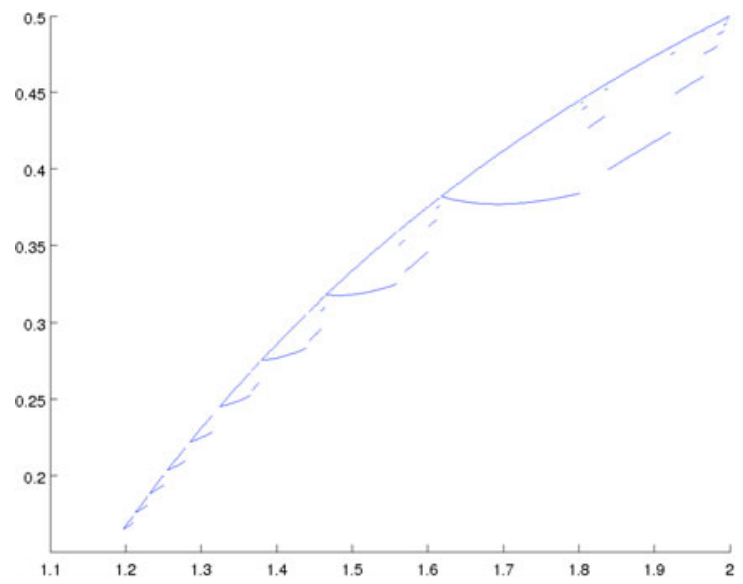

FIGURE 3. A plot of $1-(1 / \beta)$ and $1-(1 / \beta)-\left(1 / \beta^{m}\right)+\left(1 / \beta\left(\beta^{m}-1\right)\right)$ for basic intervals corresponding to Farey words of length $m$ with $m \leq 10$.

Then we have for each $\beta \in\left(\gamma_{L}, \gamma_{R}\right]$ that

$$
\begin{aligned}
t^{*} & =\pi_{\beta}\left(0 a_{2} \ldots a_{m}\left(a_{1} a_{2} \ldots a_{m}\right)^{\infty}\right) \\
& >1-\frac{1}{\beta}-\frac{1}{\beta^{m}}+\sum_{i=1}^{\infty} \frac{1}{\beta^{i m+1}}=1-\frac{1}{\beta}-\frac{1}{\beta^{m}}+\frac{1}{\beta\left(\beta^{m}-1\right)} .
\end{aligned}
$$

From Proposition 5.2 we know that $\pi_{\gamma_{L}}\left(0 a_{2} \ldots a_{m}^{+} 0^{\infty}\right)=1-\left(1 / \gamma_{L}\right)$. For $\beta>\gamma_{L}$ we have $a_{1} \ldots a_{m}^{+} 0^{\infty} \prec b(1, \beta)$, so that

$$
t^{\diamond}=\pi_{\beta}\left(0 a_{2} \ldots a_{m}^{+} 0^{\infty}\right)=\pi_{\beta}\left(a_{1} \ldots a_{m}^{+} 0^{\infty}\right)-\pi_{\beta}\left(10^{\infty}\right)<1-\frac{1}{\beta} .
$$

In Figure 3 we see a plot of the lower and upper bounds for $\tau_{\beta}$ found in Lemma 5.4.

The next lemma considers the critical point $\tau_{\beta}$ for the remaining values of $\beta$, i.e., those that are not in the closure of a Farey interval.

LEMMA 5.5. Let $\beta \in(1,2) \backslash \bigcup\left[\gamma_{L}, \gamma_{R}\right]$ with the union taken over all Farey intervals. Then $\max \overline{E_{\beta}^{+}}=\tau_{\beta}=1-(1 / \beta)$.

Proof. Take $\beta \in(1,2) \backslash \bigcup\left[\gamma_{L}, \gamma_{R}\right]$. First we show that $\tau_{\beta} \geq 1-(1 / \beta)$. Let $t<$ $1-(1 / \beta)$ with $b(t, \beta)=\left(b_{i}(t, \beta)\right)$. Since $\operatorname{dim}_{H}\left((1,2) \backslash \bigcup\left[\gamma_{L}, \gamma_{R}\right]\right)=0$ there exists a sequence of Farey intervals $\left(\left[\gamma_{L, k}, \gamma_{R, k}\right]\right)$ such that $\gamma_{L, k} \nearrow \beta$ as $k \rightarrow \infty$. Thus, as $k \rightarrow \infty$ we have

$$
\sum_{i=1}^{\infty} \frac{b_{i}(t, \beta)}{\left(\gamma_{L, k}\right)^{i}} \searrow \sum_{i=1}^{\infty} \frac{b_{i}(t, \beta)}{\beta^{i}}=t \quad \text { and } \quad 1-\frac{1}{\gamma_{L, k}} \nearrow 1-\frac{1}{\beta} .
$$

For each $k$ we have a sequence $\left(\mathbf{t}_{k, N}\right) \subseteq \mathcal{E}_{\gamma_{L, k}}^{+}$as given in (5.1). Since $\gamma_{L, k}<\beta$ we obtain for each $N, n \geq 1$ that

$$
\mathbf{t}_{k, N} \preccurlyeq \sigma^{n}\left(\mathbf{t}_{k, N}\right) \prec \alpha\left(\gamma_{L, k}\right) \prec \alpha(\beta) .
$$


Hence, $\mathbf{t}_{k, N} \in \mathcal{E}_{\beta}^{+}$for all $k \geq 1$ and $N \geq 1$. This gives that $\max \overline{E_{\beta}^{+}} \geq 1-(1 / \beta)$. Moreover, since $t<1-(1 / \beta)$ we can find by (5.5) a sufficiently large $M \in \mathbb{N}$ such that

$$
t<t_{1}:=\sum_{i=1}^{\infty} \frac{b_{i}(t, \beta)}{\left(\gamma_{L, M}\right)^{i}}<1-\frac{1}{\gamma_{L, M}}<1-\frac{1}{\beta} .
$$

Observe that $b(t, \beta)=\left(b_{i}(t, \beta)\right)$ is a $\gamma_{L, M}$-expansion of $t_{1}$, which is lexicographically less than or equal to its greedy expansion $b\left(t_{1}, \gamma_{L, M}\right)$. Then

$$
\begin{aligned}
\mathcal{K}_{\beta}^{+}(t) & =\left\{\left(x_{i}\right): b(t, \beta) \preccurlyeq \sigma^{n}\left(\left(x_{i}\right)\right) \prec \alpha(\beta) \forall n \geq 0\right\} \\
& \supseteq\left\{\left(x_{i}\right): b\left(t_{1}, \gamma_{L, M}\right) \preccurlyeq \sigma^{n}\left(\left(x_{i}\right)\right) \prec \alpha\left(\gamma_{L, M}\right) \forall n \geq 0\right\}=\mathcal{K}_{\gamma_{L, M}}^{+}\left(t_{1}\right)
\end{aligned}
$$

Since $\tau_{\gamma_{L, M}}=1-\left(1 / \gamma_{L, M}\right)>t_{1}$ by Remark 5.3 we know that $h_{\text {top }}\left(\mathcal{K}_{\gamma_{L, M}}^{+}\left(t_{1}\right)\right)>0$ and together with (5.6) we then find that $h_{\text {top }}\left(\mathcal{K}_{\beta}^{+}(t)\right)>0$, which in turn implies that $\tau_{\beta} \geq t$. Since $t<1-(1 / \beta)$ was taken arbitrarily we conclude that $\tau_{\beta} \geq 1-(1 / \beta)$.

To prove the other inequality we show that for any $t>1-(1 / \beta)$ we have $\mathcal{K}_{\beta}^{+}(t)=\emptyset$. Take $t>1-(1 / \beta)$. There is a sequence of Farey intervals $\left(\left[\gamma_{L, k}, \gamma_{R, k}\right]\right)$ such that $\gamma_{L, k} \searrow \beta$ as $k \rightarrow \infty$. Thus, when $k \rightarrow \infty$ we have

$$
\sum_{i=1}^{\infty} \frac{b_{i}(t, \beta)}{\left(\gamma_{L, k}\right)^{i}} \nearrow \sum_{i=1}^{\infty} \frac{b_{i}(t, \beta)}{\beta^{i}}=t \quad \text { and } \quad 1-\frac{1}{\gamma_{L, k}} \searrow 1-\frac{1}{\beta} .
$$

Since $t>1-(1 / \beta)$ we can find a sufficiently large $N \in \mathbb{N}$ such that

$$
1-\frac{1}{\beta}<1-\frac{1}{\gamma_{L, N}}<t_{2}:=\sum_{i=1}^{\infty} \frac{b_{i}(t, \beta)}{\left(\gamma_{L, N}\right)^{i}}<t .
$$

Since $\gamma_{L, N}>\beta, b(t, \beta)$ is the greedy $\gamma_{L, N}$-expansion of $t_{2}$, i.e., $b(t, \beta)=b\left(t_{2}, \gamma_{L, N}\right)$. Therefore,

$$
\begin{aligned}
\mathcal{K}_{\beta}^{+}(t) & \subseteq\left\{\left(x_{i}\right): b\left(t_{2}, \gamma_{L, N}\right) \preccurlyeq \sigma^{n}\left(\left(x_{i}\right)\right) \prec \alpha\left(\gamma_{L, N}\right) \forall n \geq 0\right\} \\
& =\mathcal{K}_{\gamma_{L, N}}^{+}\left(t_{2}\right) \subseteq \mathcal{K}_{\gamma_{L, N}}^{+}\left(\tau_{\gamma_{L, N}}\right) .
\end{aligned}
$$

From (5.3) we conclude that $\mathcal{K}_{\beta}^{+}(t)=\emptyset$ and hence max $\overline{E_{\beta}^{+}}, \tau_{\beta} \leq t$. Since $t>1-(1 / \beta)$ was taken arbitrarily we have $\max \overline{E_{\beta}^{+}}=\tau_{\beta}=1-(1 / \beta)$.

Proof of Theorem D. From Proposition 5.2 and Lemmas 5.4 and 5.5 we know that for all $\beta \in(1,2)$ we have $\tau_{\beta} \leq 1-(1 / \beta)$ with equality only if $\beta \in(1,2) \backslash \bigcup\left(\gamma_{L}, \gamma_{R}\right]$. We also know that for these points $\tau_{\beta}=\max \overline{E_{\beta}^{+}}$.

By Proposition 3.3 we know that any isolated point of $E_{\beta}^{+}$has a periodic greedy $\beta$-expansion $b(t, \beta)$. From Proposition 3.10 it follows that any $t \in(0,1)$, for which $b(t, \beta)=\left(s_{1} \ldots s_{m}\right)^{\infty}$ is Lyndon, is isolated in $E_{\beta}^{+}$if and only if $\beta$ lies in the basic interval associated to $\left(s_{1} \ldots s_{m}\right)^{\infty}$. Since Farey intervals are maximal by Proposition 4.7, if $\beta \notin \bigcup\left(\gamma_{L}, \gamma_{R}\right]$, then $E_{\beta}^{+}$cannot contain an isolated point and $\overline{E_{\beta}^{+}}$is a Cantor set. 


\section{Final observations and remarks}

With the results from Theorems $\mathrm{B}$ and $\mathrm{C}$ we have shown that the situation for $\beta \in(1,2)$ differs drastically from the situation for $\beta=2$ that was previously investigated in [Urb86, Nil09, CT17]. There are still several unanswered questions.

Firstly, the structure of $E_{\beta}^{0}$ remains illusive to us. We know that $t \in E_{\beta}^{0}$ is isolated in $E_{\beta}$ if $\beta-1 \notin K_{\beta}(t)$ and in Proposition 2.6 we proved that $h_{\text {top }}\left(\mathcal{K}_{\beta}(t)\right)=h_{\text {top }}\left(\mathcal{K}_{\beta}^{+}(t)\right)$ for any $t \in E_{\beta}^{+}$. It would be interesting to know whether $t \in E_{\beta}^{0}$ is isolated in $E_{\beta}$ in case $\beta-1 \in K_{\beta}(t)$ and to consider $h_{\text {top }}\left(\mathcal{K}_{\beta}^{0}(t)\right)$ also in case $t \notin E_{\beta}^{+}$.

In the previous section we have investigated the value of the critical point $\tau_{\beta}$ of the dimension function $\eta_{\beta}: t \mapsto \operatorname{dim}_{H} K_{\beta}(t)$. We could determine this value for any $\beta$ in the set $(1,2) \backslash \bigcup\left(\gamma_{L}, \gamma_{R}\right]$. If $\beta \in\left(\gamma_{L}, \gamma_{R}\right]$ for some Farey interval $\left(\gamma_{L}, \gamma_{R}\right]$, we only have a lower and an upper bound for $\tau_{\beta}$. With a calculation very similar to the one in (5.4) one can show that for any $\beta \in\left(\gamma_{L}, \gamma_{R}\right]$ that satisfies

$$
\alpha(\beta) \prec a_{1} \ldots a_{m}^{+}\left(0 a_{2} \ldots a_{m}\right)\left(a_{1} \ldots a_{m}\right)^{\infty}
$$

we have $\tau_{\beta}=t^{*}$. However, for larger values of $\beta \in\left(\gamma_{L}, \gamma_{R}\right]$ the situation seems more intricate. It would be interesting to consider this question further by specifying $\tau_{\beta}$ more precisely also on $\bigcup\left(\gamma_{L}, \gamma_{R}\right]$ and by analysing the behaviour of the function $\tau: \beta \mapsto \tau_{\beta}$.

For $\beta=2$ it is shown in [Urb86] that $\operatorname{dim}_{H}\left(E_{2} \cap[t, 1]\right)=\operatorname{dim}_{H} K_{2}(t)$. Motivated by Proposition 2.7 we conjecture the following.

Conjecture 6.1. For any $t \in[0,1)$ and any $\beta \in(1,2)$ we have $\operatorname{dim}_{H}\left(E_{\beta} \cap[t, 1]\right)=$ $\operatorname{dim}_{H} K_{\beta}(t)$.

Acknowledgements. We would like to thank the anonymous referee for valuable suggestions, especially in strengthening Proposition 4.4 and giving an easier proof. The first author was partially supported by the NWO Veni grant 639.031.140. The second author was supported by NSFC No. 11401516. The fourth author was supported by NSFC Nos. 11671147 and 11571144 and the Science and Technology Commission of Shanghai Municipality (STCSM) No. 18dz2271000.

\section{REFERENCES}

[AB14] R. Alcaraz Barrera. Topological and ergodic properties of symmetric sub-shifts. Discrete Contin. Dyn. Syst. 34(11) (2014), 4459-4486.

[BW14] Y. Bugeaud and B. Wang. Distribution of full cylinders and the Diophantine properties of the orbits in $\beta$-expansions. J. Fractal Geom. 1(2) (2014), 221-241.

[BY11] L. Bunimovich and A. Yurchenko. Where to place a hole to achieve a maximal escape rate. Israel J. Math. 182 (2011), 229-252.

[Cao14] C.-Y. Cao. A result on the approximation properties of the orbit of 1 under the $\beta$-transformation. J. Math. Anal. Appl. 420(1) (2014), 242-256.

[CIT18] C. Carminati, S. Isola and G. Tiozzo. Continued fractions with $\operatorname{SL}(2, \mathbb{Z})$-branches: combinatorics and entropy. Trans. Amer. Math. Soc. 370(7) (2018), 4927-4973.

[Cla16] L. Clark. The $\beta$-transformation with a hole. Discrete Contin. Dyn. Syst. 36(3) (2016), 1249-1269.

[CT17] C. Carminati and G. Tiozzo. The local Hölder exponent for the dimension of invariant subsets of the circle. Ergod. Th. \& Dynam. Sys. 37(6) (2017), 1825-1840. 
[Det13] C. Dettmann. Open circle maps: small hole asymptotics. Nonlinearity 26(1) (2013), 307-317.

[dVKL16] M. de Vries, V. Komornik and P. Loreti. Topology of the set of univoque bases. Topology Appl. 205 (2016), 117-137.

[GL15] Y. Ge and F. Lü. A note on inhomogeneous Diophantine approximation in beta-dynamical system. Bull. Aust. Math. Soc. 91(1) (2015), 34-40.

[GS15] P. Glendinning and N. Sidorov. The doubling map with asymmetrical holes. Ergod. Th. \& Dynam. Sys. 35(4) (2015), 1208-1228.

[KL07] V. Komornik and P. Loreti. On the topological structure of univoque sets. J. Number Theory 122(1) (2007), 157-183.

[Lot02] M. Lothaire. Algebraic Combinatorics on Words. Cambridge University Press, Cambridge, 2002.

[LPWW14] B. Li, T. Persson, B. Wang and J. Wu. Diophantine approximation of the orbit of 1 in the dynamical system of beta expansions. Math. Z. 276(3-4) (2014), 799-827.

[LW16] F. Lü and J. Wu. Diophantine analysis in beta-dynamical systems and Hausdorff dimensions. Adv. Math. 290 (2016), 919-937.

[Nil07] J. Nilsson. On numbers badly approximable by $q$-adic rationals. PhD Thesis, Lund University and Université du Sud Toulon-Var, 2007.

[Nil09] J. Nilsson. On numbers badly approximable by dyadic rationals. Israel J. Math. 171 (2009), 93-110.

[Par60] W. Parry. On the $\beta$-expansions of real numbers. Acta Math. Acad. Sci. Hungar. 11 (1960), 401-416.

[PS08] T. Persson and J. Schmeling. Dyadic Diophantine approximation and Katok's horseshoe approximation. Acta Arith. 132(3) (2008), 205-230.

[Rai89] P. Raith. Hausdorff dimension for piecewise monotonic maps. Studia Math. 94(1) (1989), 17-33.

[Rai92] P. Raith. Continuity of the Hausdorff dimension for piecewise monotonic maps. Israel J. Math. 80(1-2) (1992), 97-133.

[Rai94] P. Raith. Continuity of the Hausdorff dimension for invariant subsets of interval maps. Acta Math. Univ. Comenian. (N.S.) 63(1) (1994), 39-53.

[Rén57] A. Rényi. On algorithms for the generation of real numbers. Magyar Tud. Akad. Mat. Fiz. Oszt. Közl. 7 (1957), 265-293.

[Sch97] J. Schmeling. Symbolic dynamics for $\beta$-shifts and self-normal numbers. Ergod. Th. \& Dynam. Sys. 17(3) (1997), 675-694.

[Sid14] N. Sidorov. Supercritical holes for the doubling map. Acta Math. Hungar. 143(2) (2014), 298-312.

[SM94] R. Siromoney, L. Mathew, V. R. Dare and K. G. Subramanian. Infinite Lyndon words. Inform. Process. Lett. 50(2) (1994), 101-104.

[Urb86] M. Urbański. On Hausdorff dimension of invariant sets for expanding maps of a circle. Ergod. Th. \& Dynam. Sys. 6(2) (1986), 295-309.

[Urb87] M. Urbański. Invariant subsets of expanding mappings of the circle. Ergod. Th. \& Dynam. Sys. 7(4) (1987), 627-645. 